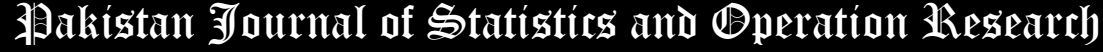

\section{A New Extreme Value Model with Different Copula, Statistical Properties and Applications}

\author{
Hanaa Elgohari ${ }^{1 *}$ and Haitham M. Yousof ${ }^{2}$
}

\author{
* Corresponding Author \\ ${ }^{1}$ Department of Applied Statistics, Faculty of Commerce, Mansoura University, Egypt, \\ hanaa_elgohary@mans.edu.eg \\ ${ }^{2}$ Department of Statistics, Mathematics and Insurance, Benha University, Benha, Egypt, \\ haitham.yousof@fcom.bu.edu.eg
}

\begin{abstract}
In this article, we defined and studied a new distribution for modeling extreme value. Some of its mathematical properties are derived and analyzed. Simple types copula is employed for proposing many bivariate and multivariate type extensions. Method of the maximum likelihood estimation is employed to estimate the model parameters. Graphically, we perform the simulation experiments to assess of the finite sample behavior of the maximum likelihood estimations. Three applications are presented for measuring the flexibility of the new model is illustrated using three real data applications.
\end{abstract}

Key Words: Fréchet; Extreme Value Theory; Estimation; Clayton Copula; Renyi's entropy; Simulation; Farlie Gumbel Morgenstern family.

Mathematical Subject Classification: 62N01; 62N02; 62E10.

\section{Introduction and motivation}

The Fréchet ( $\mathrm{Fr}$ ) model is one of the most important distributions in modeling extreme values. The Fr model was originally proposed by Fréchet (1927). It has many applications in ranging, accelerated life testing, earthquakes, the floods, the wind speeds, the horse racing, the rainfall, queues in supermarkets and sea waves. One can find more details about the Fr model in the literature for example: Nadarajah and Kotz (2003) investigated the exponentiated Fr distribution. Nadarajah and Kotz (2008) discussed the sociological models based on Fr random variables (RVs), Zaharim et al. (2009) applied the Fr distribution for analyzing the wind speed data, Barreto-Souza et al. (2011) (beta Fr distribution), Krishna et al. (2013) (Marshall--Olkin Fr distribution), Mahmoud and Mandouh (2013) (transmuted Fr distribution), Mead and Abd-Eltawab (2014) (Kumaraswamy Fr distribution), Mead et al. (2016) (beta exponential Fr distribution), among others.

In this article, we expanded the extreme value theory (EVT) theory by proposing and studying a new version of the Fr model called the generalized Odd-Burr generalized Fréchet (OB-Fr) model (for more details about EVT see Fréchet (1927) and Fisher and Tippett (1928)). The new model is derived based on compiling the standard F model with the Odd-Burr generalized (OB-G) family (see Alizadeh et al. (2016)). Straightforward types of copula are employed for proposing many bivariate $\mathrm{OB}-\mathrm{Fr}(\mathrm{BvOB}-\mathrm{Fr})$ and multivariate $\mathrm{OB}-\mathrm{Fr}$ (MOB-Fr) type extensions. A RV $T$ is said to have the Fr distribution if its probability density function (PDF) and cumulative distribution function (CDF) are given by

and

$$
\pi_{\alpha, \beta}(t)=\left.\beta \alpha^{\beta} t^{-(\beta+1)} \exp \left(-\alpha^{\beta} t^{-\beta}\right)\right|_{t \geq 0}
$$

where $\beta>0$ refers to the shape parameter.

$$
\Pi_{\alpha, \beta}(t)=\left.\exp \left(-\alpha^{\beta} t^{-\beta}\right)\right|_{t \geq 0}
$$


For $\beta=2$ we get the Inverse Rayleigh (IR) model. Due to Alizadeh et al. (2016), the CDF of the OB-G family is given by

$$
F_{a, b, \underline{r}}(t)=1-\frac{\bar{\Pi}_{\underline{r}}(t)^{a b}}{\left[\Pi_{\underline{r}}(t)^{a}+\bar{\Pi}_{\underline{r}}(t)^{a}\right]^{b}},
$$

where $\bar{\Pi}_{\underline{r}}(t)=1-\Pi_{\underline{r}}(t)$. The PDF corresponding to (3) is given by

$$
f_{a, b, \underline{r}}(t)=\frac{a b \pi_{\underline{\underline{r}}}(t) \Pi_{\underline{r}}(t)^{a-1} \bar{\Pi}_{\underline{r}}(t)^{a b-1}}{\left[\Pi_{\underline{r}}(t)^{a}+\bar{\Pi}_{\underline{r}}(t)^{a}\right]^{1+b}} .
$$

For $b=1$, the OB-G family reduces to the Odd G (O-G) family (see Gleaton and Lynch (2006)). For $a=1$, the OB-G family reduces to the Proportional reversed hazard rate family (PRHR) (see Gupta and Gupta (2007)). In this work, we define and study a new Fréchet model based on OB-G family called generalized odd log-logistic F (OB-Fr) model. The OB-Fr survival function (SF) is given by

$$
S_{\underline{P}}(t)=\frac{\left[1-\exp \left(-\alpha^{\beta} t^{-\beta}\right)\right]^{a b}}{\left\{\exp \left(-a \alpha^{\beta} t^{-\beta}\right)+\left[1-\exp \left(-\alpha^{\beta} t^{-\beta}\right)\right]^{a}\right\}^{b}},
$$

where $S_{\underline{P}}(t)=1-\left.F_{\underline{P}}(t)\right|_{(\underline{P}=a, b, \alpha, \beta)}$. For $b=1$, the OB-Fr reduces to the O-Fr. For $a=1$, the OB-Fr reduces to the PRHR-Fr. The PDF corresponding to (5) is given by

$$
f_{P}(t)=\frac{a b \beta t^{-(\beta+1)} \exp \left(-a \alpha^{\beta} t^{-\beta}\right)\left[1-\exp \left(-\alpha^{\beta} t^{-\beta}\right)\right]^{a b-1}}{\left\{\exp \left(-a \alpha^{\beta} t^{-\beta}\right)+\left[1-\exp \left(-\alpha^{\beta} t^{-\beta}\right)\right]^{a}\right\}^{1+b}} .
$$

The hazard rate function (HRF) for the new model can be derived from $f_{\underline{P}}(t) / S_{\underline{P}}(t)$. The new model in (6) can be used in modeling extreme data such as the extreme floods, maximum sizes of ecological populations, the size of freak waves, the amounts of large insurance losses, equity risks, day to day market risk, side effects of drugs (e.g., Ximelagatran), survival time data, strengths and breaking stress experiments, large wildfires and repair times data. In mathematical analysis, the asymptotic analysis is used for describing the limiting behavior of some functions. Asymptotics derivations for the CDF, PDF and HRF can be obtained for the new model. The asymptotics of the CDF, $\mathrm{PDF}$ and HRF as $t \rightarrow V$ are given by

and

$$
\left.F_{\underline{P}}(t) \sim b \exp \left(-a \alpha^{\beta} t^{-\beta}\right)\right|_{t \rightarrow 0},\left.f_{\underline{P}}(t) \sim a b \beta t^{-(\beta+1)} \exp \left(-a \alpha^{\beta} t^{-\beta}\right)\right|_{t \rightarrow 0}
$$

$$
\left.h_{\underline{P}}(t) \sim a b \beta t^{-(\beta+1)} \exp \left(-a \alpha^{\beta} t^{-\beta}\right)\right|_{t \rightarrow 0} .
$$

The asymptotics of CDF, PDF and HRF as $t \rightarrow \infty$ are given by

and

$$
\left.S_{\underline{P}}(t) \sim a^{b}\left[1-\exp \left(-\alpha^{\beta} t^{-\beta}\right)\right]^{b}\right|_{t \rightarrow \infty},\left.f_{\underline{P}}(t) \sim b a^{b} \beta \frac{t^{-(\beta+1)} \exp \left(-\alpha^{\beta} t^{-\beta}\right)}{\left[1-\exp \left(-\alpha^{\beta} t^{-\beta}\right)\right]^{1-b}}\right|_{t \rightarrow \infty}
$$

$$
\left.h_{\underline{P}}(t) \sim \frac{b \beta t^{-(\beta+1)} \exp \left(-\alpha^{\beta} t^{-\beta}\right)}{1-\exp \left(-\alpha^{\beta} t^{-\beta}\right)}\right|_{t \rightarrow \infty}
$$

For simulation of this new model, we obtain the quantile function (QF) of $T$ (by inverting (5)), say $t_{u}=F^{-1}(u)$, as

$$
t_{u}=\left\{-\frac{1}{\alpha^{\beta}} \ln \left[\frac{\left(1-\bar{u}^{\frac{1}{b}}\right)^{\frac{1}{a}}}{\bar{u}^{\frac{1}{a b}}+\left(1-\bar{u}^{\frac{1}{b}}\right)^{\frac{1}{a}}}\right]\right\}^{-\frac{1}{\beta}},
$$

where $\bar{u}=1-u$. Equation (7) is used for simulating the new model (see Section 4). Many useful Fréchet extension can be cited and used in future comparisons see, for example, Korkmaz et al. (2017), Yousof et al. (2017), Haq et al. (2017), Korkmaz et al. (2017), Yousof et al. (2018b), Jahanshahi et al. (2019), Yousof et al. (2019a, b), Salah et al. (2020), Al-Babtain et al. (2020), Elsayed and Yousof (2020), Ibrahim et al. (2020) and

Yousof et al. (2020), Ibrahim et al. (2021), among others

\section{Copula}

In this section, we derive some new bivariate $\mathrm{OB}-\mathrm{Fr}(\mathrm{BvOB}-\mathrm{Fr})$ via

i. $\quad$ Farlie Gumbel Morgenstern (FGM) Copula. 


\section{ii. $\quad$ Modified Farlie Gumbel Morgenstern (FGM) Copula. \\ iii. Clayton Copula. \\ iv. Renyi's entropy.}

The Multivariate OB-Fr (MOB-Fr) type is also presented. However, future works may be allocated to study these new models. For more details see Ali et al. (2021a, b), Elgohari and Yousof (2020b and 2021) and Shehata and Yousof (2021a, b).

\subsection{FGM Copula}

Consider the joint CDF of the FGM family (see Morgenstern (1956), Gumbel (1958) and Gumbel (1960)), then $C_{\sigma}(u, v)=u v(1+\sigma \overline{u v})$ where the marginal function $u=F_{1}\left(t_{1}\right), \quad v=F_{2}\left(t_{2}\right) \in(0,1), \quad \sigma \in(-1,1)$ is a dependence parameter and for every $u, v \in(0,1), C_{\sigma}(u, 0)=C_{\sigma}(0, v)=0$ which is "grounded minimum" and $C_{\sigma}(u, 1)=u$ and $C_{\sigma}(1, v)=v$ which is "grounded maximum". Then, setting

$$
\bar{u}=\bar{u}_{\underline{P}_{1}}=\left.\frac{\left(1-\boldsymbol{Q}_{\alpha, \beta_{1}, t_{1}}\right)^{a_{1} b_{1}}}{\left[\boldsymbol{Q}_{\alpha, \beta_{1}, t_{1}}^{a_{1}}+\left(1-\boldsymbol{Q}_{\alpha, \beta_{1}, t_{1}}\right)^{a_{1}}\right]^{b_{1}}}\right|_{\underline{P}_{1}=a_{1}, b_{1}, \beta_{1}>0},
$$

where $\boldsymbol{Q}_{\alpha, \beta_{1}, t_{1}}=\exp \left(-\alpha^{\beta_{1}} t_{1}^{-\beta_{1}}\right)$ and

$$
\bar{v}=\bar{v}_{\underline{P}_{2}}=1-\left.\frac{\left[1-\boldsymbol{Q}_{\alpha, \beta_{2}, t_{2}}\right]^{a_{2} b_{2}}}{\left\{\boldsymbol{Q}_{\alpha, \beta_{2}, t_{2}}^{a_{2}}+\left[1-\boldsymbol{Q}_{\alpha, \beta_{2}, t_{2}}\right]^{a_{2}}\right\}^{b_{2}}}\right|_{\underline{P}_{2}=a_{2}, b_{2}, \beta_{2}>0},
$$

where $\boldsymbol{Q}_{\alpha, \beta_{2}, t_{2}}=\exp \left(-\alpha^{\beta_{2}} t_{2}^{-\beta_{2}}\right)$ then we have

$$
\begin{gathered}
F\left(t_{1}, t_{2}\right)=\left.C\left(F_{\underline{P}_{1}}\left(t_{1}\right), F_{\underline{P}_{2}}\left(t_{2}\right)\right)\right|_{\left(\alpha=\alpha_{1}=\alpha_{2}\right)}=\left\{1-\frac{\left(1-\boldsymbol{Q}_{\alpha, \beta_{1}, t_{1}}\right)^{a_{1} b_{1}}}{\left[\boldsymbol{Q}_{\alpha, \beta_{1}, t_{1}}^{a_{1}}+\left[1-\boldsymbol{Q}_{\alpha, \beta_{1}, t_{1}}\right]^{a_{1}}\right]^{b_{1}}}\right\} \\
\times\left\{1-\frac{\left(1-\boldsymbol{Q}_{\alpha, \beta_{2}, t_{2}}\right)^{a_{2} b_{2}}}{\left[\boldsymbol{Q}_{\alpha, \beta_{2}, t_{2}}^{a_{2}}+\left(1-\boldsymbol{Q}_{\alpha, \beta_{2}, t_{2}}\right)^{a_{2}}\right]^{b_{2}}}\right\}\left(1+\sigma\left\{\begin{array}{c}
\frac{\left(1-\boldsymbol{Q}_{\alpha, \beta_{1}, t_{1}}\right)^{a_{1} b_{1}}}{\left[\boldsymbol{Q}_{\alpha, \beta_{1}, t_{1}}^{a_{1}}+\left[1-\boldsymbol{Q}_{\alpha, \beta_{1}, t_{1}}\right]^{a_{1}}\right]^{b_{1}}} \\
\times \frac{\left(1-\boldsymbol{Q}_{\alpha, \beta_{2}, t_{2}}\right)^{a_{2} b_{2}}}{\left[\boldsymbol{Q}_{\alpha, \beta_{2}, t_{2}}^{a_{2}}+\left(1-\boldsymbol{Q}_{\alpha, \beta_{2}, t_{2}}\right)^{a_{2}}\right]^{b_{2}}}
\end{array}\right\} .\right.
\end{gathered}
$$

The joint PDF can then be derived from

$$
c_{\sigma}(u, v)=1+\left.\sigma u^{*} v^{*}\right|_{\left(u^{*}=1-2 u \text { and } v^{*}=1-2 v\right)}
$$

or from

$$
f\left(t_{1}, t_{2}\right)=\left.f_{\underline{P_{1}}}\left(t_{1}\right) f_{\underline{P}_{2}}\left(t_{2}\right) c\left(F_{\underline{P}_{1}}\left(t_{1}\right), F_{P_{2}}\left(t_{2}\right)\right)\right|_{\left(\alpha=\alpha_{1}=\alpha_{2}\right)} .
$$

\subsection{Modified FGM Copula}

Consider the following modified version of the bivariate FGM copula defined as (see Rodriguez-Lallena and UbedaFlores (2004))

$$
\left.C_{\sigma}(u, v)\right|_{\sigma \in[-1,1]}=u v[1+\sigma \Phi(u) \Psi(v)]=u v+\sigma \Phi \cdot(u) \Psi \cdot(v),
$$

where $\Phi \cdot(u)=u \Phi(u)$, and $\Psi \cdot(v)=v \Psi(v)$. Where $\Phi(u)$ and $\Psi(v)$ are two absolutely continuous functions on $(0,1)$ with the following conditions:

1-The boundary condition:

$$
\Phi(0)=\Phi(1)=\Psi(0)=\Psi(1)=0
$$

2-Let

$$
\begin{aligned}
& \alpha=\inf \left\{\frac{\partial}{\partial u} \Phi(u): A_{1}\right\}<0, \beta=\sup \left\{\frac{\partial}{\partial u} \Phi(u): A_{1}\right\}<0, \\
& \xi=\inf \left\{\frac{\partial}{\partial v} \Psi \cdot(v): A_{2}\right\}>0, \eta=\sup \left\{\frac{\partial}{\partial v} \Psi \cdot(v): A_{2}\right\}>0,
\end{aligned}
$$


Then, $\min (\alpha \beta, \xi \eta) \geq 1$, where

and

$$
\begin{gathered}
\frac{\partial}{\partial u} \Phi(u)=\Phi(u)+u \frac{\partial}{\partial u} \Phi(u), \\
A_{1}=\left\{u: u \in(0,1) \mid \frac{\partial}{\partial u} \Phi(u) \text { exists }\right\},
\end{gathered}
$$

$$
A_{2}=\left\{v: v \in(0,1) \mid \frac{\partial}{\partial v} \Psi \cdot(v) \text { exists }\right\}
$$

\section{BvOB-Fr-FGM (Type I) model}

Here, we consider the following functional form for both $\Phi(u)$ and $\Psi(v)$ as

$$
C_{\sigma}(u, v)=\sigma[\Phi \cdot(u) \Psi \cdot(v)]+\left\{1-\frac{\left(1-\boldsymbol{Q}_{\alpha, \beta_{1}, u}\right)^{a_{1} b_{1}}}{\left[\boldsymbol{Q}_{\alpha, \beta_{1}, u}^{a_{1}}+\left(1-\boldsymbol{Q}_{\alpha, \beta_{1}, u}\right)^{a_{1}}\right]^{b_{1}}}\right\}\left\{1-\frac{\left[1-\boldsymbol{Q}_{\alpha, \beta_{2}, v}\right]^{a_{2} b_{2}}}{\left\{\boldsymbol{Q}_{\alpha, \beta_{2}, v}^{a_{2}}+\left[1-\boldsymbol{Q}_{\alpha, \beta_{2}, v}\right]^{a_{2}}\right\}^{b_{2}}}\right\},
$$

where

and

$$
\Phi(u)=\left.\frac{u\left(1-\mathcal{Q}_{\alpha, \beta_{1}, u}\right)^{a_{1} b_{1}}}{\left[\boldsymbol{Q}_{\alpha, \beta_{1}, u}^{a_{1}}+\left(1-\mathcal{Q}_{\alpha, \beta_{1}, u}\right)^{a_{1}}\right]^{b_{1}}}\right|_{\left(a_{1}, b_{1}, \beta_{1}\right)>0}
$$

$$
\Psi \cdot(v)=\left.\frac{v\left[1-\boldsymbol{Q}_{\alpha, \beta_{2}, v}\right]^{a_{2} b_{2}}}{\left\{\boldsymbol{Q}_{\alpha, \beta_{2}, v}^{a_{2}}+\left[1-\boldsymbol{Q}_{\alpha, \beta_{2}, v}\right]^{a_{2}}\right\}^{b_{2}}}\right|_{\left(a_{2}, b_{2}, \beta_{2}\right)>0} .
$$

\section{BvOB-Fr-FGM (Type II) model}

Consider the following functional form for both $\Phi(u)$ and $\Psi(v)$ which satisfy all the conditions stated earlier where

$$
\left.\Phi(u)\right|_{\left(\sigma_{1}>0\right)}=u^{\sigma_{1}}(1-u)^{1-\sigma_{1}} \text { and }\left.\Psi(v)\right|_{\left(\sigma_{2}>0\right)}=v^{\sigma_{2}}(1-v)^{1-\sigma_{2}} .
$$

The corresponding bivariate copula (henceforth, BvOB-Fr-FGM (Type II) copula) can be derived from

$$
C_{\sigma, \sigma_{1}, \sigma_{2}}(u, v)=u v\left[1+\sigma u^{\sigma_{1}} v^{\sigma_{2}}(1-u)^{1-\sigma_{1}}(1-v)^{1-\sigma_{2}}\right] .
$$

\section{BvOB-Fr-FGM (Type III) model}

Consider the following functional form for both $\Phi(u)$ and $\Psi(v)$ which satisfy all the conditions stated earlier where

$$
P(u)=u[\log (1+\bar{u})] \text { and } Q(v)=v[\log (1+\bar{v})] .
$$

In this case, one can also derive a closed form expression for the associated CDF of the BvOB-Fr-FGM (Type III) as

$$
C_{\nabla}(u, v)=u v(1+\sigma P(u) Q(v)) .
$$

\section{BvOB-Fr-FGM (Type IV) model}

Due to Ghosh and Ray (2016) the CDF of the BvOB-Fr-FGM (Type IV) model can be derived from

where

$$
C_{\sigma}(u, v)=u F^{-1}(v)+v F^{-1}(u)-F^{-1}(u) F^{-1}(v) \text {, }
$$

$$
F^{-1}(u)=\left(-\frac{1}{\alpha_{1}^{\beta_{1}}} \ln \left\{\frac{\left[1-(1-u)^{\frac{1}{b_{1}}}\right]^{\frac{1}{a_{1}}}}{(1-u)^{\frac{1}{a_{1} b_{1}}}+\left[1-(1-u)^{\frac{1}{b_{1}}}\right]^{\frac{1}{a_{1}}}}\right\}\right)^{-\frac{1}{\beta_{1}}},
$$

and 


$$
F^{-1}(v)=\left(-\frac{1}{\alpha_{2}^{\beta_{2}}} \ln \left\{\frac{\left[1-(1-v)^{\frac{1}{b_{2}}}\right]^{\frac{1}{a_{2}}}}{(1-v)^{\frac{1}{a_{2} b_{2}}}+\left[1-(1-v)^{\frac{1}{b_{2}}}\right]^{\frac{1}{a_{2}}}}\right\}\right)^{-\frac{1}{\beta_{2}}} .
$$

\subsection{Clayton Copula}

The Clayton Copula can be considered as

$$
C\left(v_{1}, v_{2}\right)=\left.\left(v_{1}^{-\nabla}+v_{2}^{-\nabla}-1\right)^{-\frac{1}{\nabla}}\right|_{\nabla \in[0, \infty]} .
$$

Let us assume that $T \sim \mathrm{OB}-\mathrm{Fr}\left(a_{1}, b_{1}, \beta_{1}\right)$ and $W \sim \mathrm{OB}-\mathrm{Fr}\left(a_{2}, b_{2}, \beta_{2}\right)$. Then, setting

$$
v_{1}=v(t)=1-\left.\frac{\left(1-\boldsymbol{Q}_{\alpha, \beta_{1}, t_{1}}\right)^{a_{1} b_{1}}}{\left[\boldsymbol{Q}_{\alpha, \beta_{1}, t_{1}}^{a_{1}}+\left(1-\boldsymbol{Q}_{\alpha, \beta_{1}, t_{1}}\right)^{a_{1}}\right]^{b_{1}}}\right|_{{\underline{P_{1}}}_{1},},
$$

and

$$
v_{2}=v(w)=1-\left.\frac{\left(1-\boldsymbol{Q}_{\alpha, \beta_{2}, w}\right)^{a_{2} b_{2}}}{\left[\boldsymbol{Q}_{\alpha, \beta_{2}, w}^{a_{2}}+\left(1-\boldsymbol{Q}_{\alpha, \beta_{2}, w}\right)^{a_{2}}\right]^{b_{2}}}\right|_{{\underline{P_{2}}}_{2}>0},
$$

Then, the BvOB-Fr type distribution can be derived as

$$
F(t, w)=\left.C\left(F_{P_{1}}(t), F_{\underline{P}_{2}}(w)\right)\right|_{\left(\alpha=\alpha_{1}=\alpha_{2}\right)}=\left[\begin{array}{c}
\left(1-\frac{\left(1-\mathcal{Q}_{\alpha, \beta_{1}, t_{1}}\right)^{a_{1} b_{1}}}{\left[\boldsymbol{Q}_{\alpha, \beta_{1}, t_{1}}^{a_{1}}+\left(1-\boldsymbol{Q}_{\alpha, \beta_{1}, t_{1}}\right)^{a_{1}}\right]^{b_{1}}}\right)^{-\nabla} \\
+\left(1-\frac{\left(1-\boldsymbol{Q}_{\alpha, \beta_{2}, w}\right)^{a_{2} b_{2}}}{\left[\boldsymbol{Q}_{\alpha, \beta_{2}, w}^{a_{2}}+\left(1-\boldsymbol{Q}_{\alpha, \beta_{2}, w}\right)^{a_{2}}\right]^{b_{2}}}\right)^{-\nabla} \\
-1
\end{array}\right]^{-\frac{1}{\nabla}} .
$$

\subsection{Renyi's entropy Copula}

Consider theorem of Pougaza and Djafari (2011) where then, the associated BvOB-Fr will be

$$
R(u, v)=t_{2} u+t_{1} v-t_{1} t_{2}
$$

$$
\begin{aligned}
& \left.R\left(t_{1}, t_{2}\right)\right|_{\left(\alpha=\alpha_{1}=\alpha_{2}\right)}=R\left(F_{\underline{P}_{1}}(t), F_{P_{2}}(w)\right)=-t_{1} t_{2} \\
& +t_{2}\left\{1-\frac{\left(1-\boldsymbol{Q}_{\alpha, \beta_{1}, t_{1}}\right)^{a_{1} b_{1}}}{\left[\boldsymbol{Q}_{\alpha, \beta_{1}, t_{1}}^{a_{1}}+\left[1-\boldsymbol{Q}_{\alpha, \beta_{1}, t_{1}}\right]^{a_{1}}\right]^{b_{1}}}\right\}+t_{1}\left\{1-\frac{\left(1-\boldsymbol{Q}_{\alpha, \beta_{2}, t_{2}}\right)^{a_{2} b_{2}}}{\left[\boldsymbol{Q}_{\alpha, \beta_{2}, t_{2}}^{a_{2}}+\left[1-\boldsymbol{Q}_{\alpha, \beta_{2}, t_{2}}\right]^{a_{2}}\right]^{b_{2}}}\right\} .
\end{aligned}
$$

By fixing $a$ and $b$ we then have a 5-dimension parameter BvOB-Fr type distribution.

MOB-Fr extension via Clayton Copula. A straightforward $\hbar$-dimensional extension from the above will be

Then, the MOB-Fr extension can expressed as

$$
H\left(v_{i}\right)=\left[\sum_{i=1}^{\hbar} v_{i}^{-\nabla}+1-\hbar\right]^{-\frac{1}{\nabla}}
$$

$$
\left.H(\underline{T})\right|_{\left(\alpha=\alpha_{i}\right)}=\left[\sum_{i=1}^{\hbar}\left(1-\frac{\left[1-\exp \left(-\alpha^{\beta_{i}} t_{i}^{-\beta_{i}}\right)\right]^{a_{i} b_{i}}}{\left\{\exp \left(-a_{i} t_{i}^{-\beta_{i}}\right)+\left[1-\exp \left(-t_{i}^{-\beta_{i}}\right)\right]^{a_{1}}\right\}^{b_{i}}}\right)^{-\nabla}+1-\hbar\right]^{-\frac{1}{\nabla}},
$$

where $\underline{T}=t_{1}, t_{2}, \cdots, t_{\hbar}$. 


\section{Mathematical properties}

\subsection{Useful representations}

Due to Alizadeh et al. (2016), the PDF in (6) can be expressed as

where

$$
f(t)=\sum_{s=0}^{\infty} \quad \vartheta_{s} \pi_{(1+s)}(t ; \alpha, \beta),
$$

$$
\vartheta_{s}=\frac{a b}{1+s} \sum_{d_{1}, d_{2}=0}^{\infty} \sum_{d_{3}=s}^{\infty}(-1)^{d_{2}+k+s}\left(\begin{array}{c}
-(1+b) \\
d_{1}
\end{array}\right)\left(\begin{array}{c}
-\left[a\left(1+d_{1}\right)+1\right] \\
d_{2}
\end{array}\right)\left(\begin{array}{c}
a\left(1+d_{1}\right)+d_{2}+1 \\
d_{3}
\end{array}\right)\left(\begin{array}{c}
d_{3} \\
s
\end{array}\right)
$$

and $\pi_{(1+s)}(t ; \alpha, \beta)$ is the PDF of the F model with scale parameter $\frac{\alpha}{(1+s)^{-\beta^{-1}}}$ and shape parameter $\beta$. By integrating Equation (8), the CDF of $t$ becomes

$$
F(t)=\sum_{s=0}^{\infty} \quad \vartheta_{s} \Pi_{(1+s)}(t ; \alpha, \beta),
$$

where $\Pi_{(1+s)}(t ; \alpha, \beta)$ is the $\mathrm{CDF}$ of the $\mathrm{F}$ distribution with scale parameter $\frac{\alpha}{(1+s)^{-\beta^{-1}}}$ and shape parameter $\beta$.

\subsection{Moments and incomplete moments}

The $\rho^{t h}$ ordinary moment of $T$ is given by

then we obtain

$$
\mu_{\rho, T}^{\prime}=E\left(T^{\rho}\right)=\int_{-\infty}^{\infty} t^{\rho} f(t) d t
$$

$$
\left.\mu_{\rho, T}^{\prime}\right|_{(\beta>\rho)}=\sum_{s=0}^{\infty} \alpha^{\rho} \vartheta_{s}^{(\rho, \beta)} \Gamma\left(1-\frac{\rho}{\beta}\right),
$$

where $\vartheta_{s}^{(\rho, \beta)}=\vartheta_{s}(1+s)^{\frac{\rho}{\beta}}$ and $\left.\Gamma(1+v)\right|_{\left(v \in R^{+}\right)}=v !=\prod_{\hbar=0}^{v-1} \quad(v-\hbar)$. Setting $\rho=1,2,3$ and 4 in (10), we have

$$
\begin{gathered}
\left.E(T)\right|_{(\beta>1)}=\mu_{1, T}^{\prime}=\sum_{s=0}^{\infty} \alpha \vartheta_{s}^{(1, \beta)} \Gamma\left(1-\frac{1}{\beta}\right),\left.E\left(T^{2}\right)\right|_{(\beta>2)}=\mu_{2, T}^{\prime}=\sum_{s=0}^{\infty} \alpha^{2} \vartheta_{s}^{(2, \beta)} \Gamma\left(1-\frac{2}{\beta}\right), \\
\left.E\left(T^{3}\right)\right|_{(\beta>3)}=\mu_{3, T}^{\prime}=\sum_{s=0}^{\infty} \alpha^{3} \vartheta_{s}^{(3, \beta)} \Gamma\left(1-\frac{3}{\beta}\right),
\end{gathered}
$$

and

$$
\left.E\left(T^{4}\right)\right|_{(\beta>4)}=\mu_{4, T}^{\prime}=\sum_{s=0}^{\infty} \alpha^{4} \vartheta_{s}^{(4, \beta)} \Gamma\left(1-\frac{4}{\beta}\right),
$$

where $E(T)=\mu_{1}^{\prime}$ is the mean of $T$. The $\rho^{t h}$ incomplete moment, say $I_{\rho, T}(\tau)$, of $T$ can be expressed, from (9), as

then

$$
I_{\rho, T}(\tau)=\int_{-\infty}^{\tau} t^{\rho} f(t) d t=\sum_{s=0}^{\infty} \vartheta_{s} \int_{-\infty}^{\tau} t^{\rho} \pi_{(1+s)}(t ; \beta) d t
$$

where $\gamma(\nu, \rho)$ is the incomplete gamma function.

$$
\left.I_{\rho, T}(\tau)\right|_{(\beta>\rho)}=\sum_{s=0}^{\infty} \quad \alpha^{\rho} \vartheta_{s}^{(\rho, \beta)} \gamma\left(1-\frac{\rho}{\beta},(1+s)(a / \tau)^{\beta}\right),
$$

$$
\left.\gamma(v, \rho)\right|_{(v \neq 0,-1,-2, \ldots)}=\int_{0}^{\rho} t^{v-1} \exp (-t) d t=\frac{1}{v} \rho^{v}\left\{1 F_{1}[v ; v+1 ;-\rho]\right\}=\sum_{s=0}^{\infty} \rho^{v+s} \frac{(-1)^{s}}{s !(v+s)^{\prime}},
$$

and $1 F_{1}[\because, \cdot]$ is a confluent hypergeometric function. The first incomplete moment given by (11) with $\rho=1$ as

$$
\left.I_{1, T}(\tau)\right|_{(\beta>1)}=\sum_{s=0}^{\infty} \alpha \vartheta_{s}^{(1, \beta)} \Gamma\left(1-\frac{1}{\beta},(1+s)(a / \tau)^{\beta}\right) .
$$

\subsection{Index of dispersion (Index)}

The Index of dispersion IxDis or the variance to mean ratio can derived as

$$
\operatorname{IxDis}(T)=\mu_{2} / \mu_{1}^{\prime} \text {. }
$$

It is a measure used to quantify whether a set of observed occurrences are clustered or dispersed compared to a standard statistical model. So, it indicates whether a certain statistical model is suitable for over (or under) dispersed datasets 
and is used widely in ecology as a standard measure for measuring clustering (over-dispersion) or repulsion (underdispersion). Thus, the measure can be used to assess whether observed data can be modeled using a Poisson process. For any real dataset, when the IxDis is less than 1 , the dataset is said to be "under-dispersed", this important condition can relate to occurrence patterns that are more regular than the randomness associated with a Poisson process.

\subsection{Numerical analysis}

A numerical analysis for some measures including the Mean $[E(T)]$, variance $[\operatorname{Var}(T)]$, skewness $[\operatorname{Skew}(T)]$, kurtosis $[\operatorname{Kur}(T)]$ and IxDis $(T)$ for the OB-Fr model is presented in Table 1 with useful comments. The same analysis for the standard Fr model is given in Table 2. Based on Tables 1 and 2 we note that, the Skew $(T)$ of the OBFr distribution can range in the interval $(4.5,1097)$, whereas the Skew $(Y)$ of the F model varies only in the interval $(1.2115,3.5)$. Further, the spread for the Kur $(T)$ of the OB-Fr model is ranging from 25.16 to 1354275 , whereas the spread for the Kur $(T)$ of the F model only varies from 4.5 to 98.8 with the above parameter values. The IxDis $(T)$ for the OB-Fr model can be only more 1 so it may be used as an "over-dispersed" model. Howevere the IxDis $(T)$ for the Fr model can be only between 0 and 1 so it may be used as an "under-dispersed" model.

Table 1: $\mathrm{E}(\mathrm{T}), \operatorname{Var}(\mathrm{T})$, Skew(T) and $\operatorname{Kur}(\mathrm{T})$ of the OB-Fr model.

\begin{tabular}{|c|c|c|c|c|c|c|c|c|}
\hline $\mathrm{a}$ & b & $\alpha$ & $\beta$ & $E(T)$ & $\operatorname{Var}(\mathrm{T})$ & Skew(T) & $\operatorname{Kur}(\mathrm{T})$ & $\operatorname{IxDis}(\mathrm{T})$ \\
\hline 0.00001 & & & & 0.3749728 & 18748.37 & 486.8805 & 266686 & 49999.28 \\
\hline 0.0001 & 2 & 1.5 & 1.5 & 3.747167 & 187336.7 & 154.0108 & 26686.1 & 49994.22 \\
\hline 0.001 & & & & 37.21382 & 1858581 & 48.84929 & 2686.37 & 49943.30 \\
\hline 0.01 & & & & 344.6265 & 17033731 & 15.98947 & 289.515 & 49426.64 \\
\hline 0.1 & & & & 812.7468 & 35212441 & 10.58003 & 130.309 & 43325.23 \\
\hline 0.15 & & & & 384.5686 & 14749798 & 15.93518 & 299.182 & 38354.14 \\
\hline \multirow[t]{6}{*}{0.5} & 0.00001 & 3 & 2 & 0.9996124 & 49991.05 & 298.1656 & 100016.3 & 50010.43 \\
\hline & 0.025 & & & 1975.681 & 93657162 & 6.524823 & 49.68264 & 47405.01 \\
\hline & 0.1 & & & 3919.6700 & 17038598 & 4.544012 & 25.16457 & 43469.47 \\
\hline & 0.2 & & & 3111.3620 & 12872279 & 5.249075 & 33.35269 & 41371.84 \\
\hline & 0.3 & & & 1880.6930 & 74046202 & 7.000663 & 58.60794 & 39371.77 \\
\hline & 0.4 & & & 1030.2170 & 37730469 & 9.792836 & 114.3752 & 36623.80 \\
\hline \multirow[t]{8}{*}{0.01} & 0.01 & 0.01 & 0.01 & 0.07390783 & 3688.67 & 1097.012 & 1354275 & 49909.05 \\
\hline & & 0.1 & & 0.07453191 & 3719.683 & 1092.416 & 1342959 & 49907.26 \\
\hline & & 0.2 & & 0.07472321 & 3729.19 & 1091.019 & 1339529 & 49906.72 \\
\hline & & 0.5 & & 0.07497858 & 3741.881 & 1089.162 & 1334976 & 49906.00 \\
\hline & & 10 & & 0.07583364 & 3784.372 & 1083.013 & 1319955 & 49903.61 \\
\hline & & 50 & & 0.07630605 & 3807.848 & 1079.66 & 1311801 & 49902.30 \\
\hline & & 100 & & 0.07651238 & 3818.101 & 1078.205 & 1308271 & 49901.74 \\
\hline & & 500 & & 0.07699826 & 3842.246 & 1074.803 & 1300032 & 49900.42 \\
\hline \multirow[t]{8}{*}{2} & 2 & 2 & 0.001 & 89.6136 & 4473301 & 31.45153 & 1114.489 & 49917.67 \\
\hline & & & 0.1 & 1242.69 & 4749234 & 8.732295 & 90.96676 & 38217.26 \\
\hline & & & 0.2 & 81.7061 & 1528125 & 43.42091 & 2399.856 & 18702.70 \\
\hline & & & 0.5 & 3.72747 & 84.5209 & 1050.910 & 5681077 & 22.67513 \\
\hline & & & 0.55 & 3.36541 & 32.9263 & 679.0338 & 5226987 & 9.783743 \\
\hline & & & 0.65 & 2.93612 & 9.98449 & 123.4382 & 1133658 & 3.400577 \\
\hline & & & 0.7 & 2.80062 & 6.72716 & 47.69959 & 359970.1 & 2.402026 \\
\hline & & & 0.725 & 2.74496 & 5.68505 & 30.85292 & 192929.7 & 2.071087 \\
\hline
\end{tabular}

Table 2: $\mathrm{E}(\mathrm{T}), \operatorname{Var}(\mathrm{T}), \operatorname{Skew}(\mathrm{T})$ and $\operatorname{Kur}(\mathrm{T})$ of the Fr model.

\begin{tabular}{ccccccc}
\hline$\alpha$ & $\beta$ & $\mathrm{E}(\mathrm{T})$ & $\operatorname{Var}(\mathrm{T})$ & $\mathrm{Skew}(\mathrm{T})$ & $\operatorname{Kur}(\mathrm{T})$ & $\operatorname{IxDis}(\mathrm{T})$ \\
\hline 0.5 & 5 & 0.582115 & 0.0334404 & 3.535071 & 48.0915 & 0.057446320 \\
& 10 & 0.534314 & 0.0055656 & 1.910339 & 10.9774 & 0.010416350 \\
& 25 & 0.512366 & 0.0007351 & 1.400443 & 6.85310 & 0.001434654 \\
& 40 & 0.507532 & 0.0002750 & 1.296997 & 6.23002 & 0.000541922 \\
& 50 & 0.505974 & 0.0001736 & 1.264099 & 6.04447 & 0.000343082 \\
& 75 & 0.5039371 & $7.575362 \times 10^{-05}$ & 1.221375 & 5.81425 & 0.000150324 \\
& 85 & 0.5034646 & $5.87275 \times 10^{-05}$ & 1.211504 & 5.561893 & 0.000116647
\end{tabular}




\begin{tabular}{ccccccc}
2.5 & 10 & 2.671572 & 0.1391401 & 1.910339 & 10.97857 & 0.05208173 \\
& 30 & 2.550936 & 0.0125170 & 1.353565 & 6.562312 & 0.00490683 \\
& 50 & 2.529868 & 0.0043398 & 1.264099 & 6.045233 & 0.00171541 \\
& 75 & 2.519686 & 0.0018938 & 1.221761 & 5.760403 & 0.00075161 \\
& 100 & 1.508808 & 0.0003799 & 1.216101 & 4.525062 & 0.00025185 \\
3.5 & 7.5 & 3.828837 & 0.512812 & 2.25012 & 14.9701 & 0.1339342 \\
5 & 4.5 & 5.950756 & 4.60640 & 4.23885 & 98.8016 & 0.7740870 \\
15 & 15 & 15.6475 & 1.993072 & 1.60525 & 8.282494 & 0.1273732 \\
10 & 7.5 & 10.97054 & 4.47131 & 2.29491 & 15.5896 & 0.4075740 \\
5.5 & 5.5 & 6.294259 & 3.07532 & 3.10146 & 32.22113 & 0.4885905 \\
60 & 20 & 61.8872 & 17.0379 & 1.47388 & 7.33349 & 0.2753061 \\
10 & 10 & 10.68629 & 2.226241 & 1.91034 & 10.97857 & 0.2083269 \\
\hline
\end{tabular}

\subsection{Some generating functions (GF)}

The moment generating function (MGF) can be derived using (8) as

$$
M_{T}(\tau)=\sum_{s=0}^{\infty} \vartheta_{s} M_{(1+s)}(\tau ; \beta),
$$

where $M_{(1+s)}(\tau ; \alpha, \beta)$ is the MGF of the F model with scale parameter $\frac{\alpha}{(1+s)^{-\beta^{-1}}}$ and shape parameter $\beta$, then

$$
\left.M_{T}(\tau)\right|_{(\beta>\rho)}=\sum_{s=0}^{\infty} \sum_{\rho=0}^{\infty} \alpha^{\rho} \frac{\tau^{\rho}}{\rho !} \vartheta_{s}^{(\rho, \beta)} \Gamma\left(1-\frac{\rho}{\beta}\right) .
$$

The first $\rho$ derivatives of $M_{T}(\tau)$, with respect to $\tau$ at $\tau=0$, yield the first $\rho$ moments about the origin, i.e.,

$$
\mu_{\rho, T}^{\prime}=E\left(T^{r}\right)=\left.\frac{d^{\rho}}{d \tau^{\rho}} M_{T}(\tau)\right|_{(\tau=0 \text { and } \rho=1,2,3, \ldots)},
$$

The generating function GF (CGF) is the logarithm of the MGF. Thus, $\mathrm{r}$ th cumulant, say $\kappa_{\rho}$, can be obtained from

$$
\kappa_{\rho, T}=\left.\frac{d^{\rho}}{d \tau^{\rho}} \log \left[\sum_{s=0}^{\infty} \sum_{\rho=0}^{\infty} \alpha^{\rho} \frac{\tau^{\rho}}{\rho !} \vartheta_{s}^{(\rho, \beta)} \Gamma\left(1-\frac{\rho}{\beta}\right)\right]\right|_{(\tau=0, \text { and } \rho=1,2,3, \ldots)} .
$$

The $1^{\text {st }}$ cumulant is the mean $\left(\kappa_{1}=\mu_{1}^{\prime}\right)$, the 2 nd cumulant is the variance, and the $3 r d$ cumulant is the same as the $3^{\text {rd }}$ central moment $\kappa_{3}=\mu_{3}$. But 4 th and higher order cumulants are not equal to central moments, that being said

and

$$
\begin{gathered}
\kappa_{1, T}=\mu_{1, T}^{\prime}, \\
\kappa_{2, T}=\mu_{2, T}^{\prime}-\mu_{1, T}^{\prime 2}=\mu_{2, T},
\end{gathered}
$$

$$
\kappa_{3, T}=\mu_{3, T}^{\prime}-3 \mu_{2, T}^{\prime} \mu_{1, T}^{\prime}+2 \mu_{1, T}^{\prime 3}=\mu_{3, T} .
$$

In some cases, theoretical treatments of problems in terms of cumulants are simpler than those using moments. In particular, when two or more RVs are statistically independent, the $\rho^{\text {th }}$ order cumulant of their sum is equal to the sum of their $\rho^{\text {th }}$ order cumulants. Moreover, the cumulants can be also obtained from

$$
\left.\kappa_{\rho, T}\right|_{\rho \geq 1}=\mu_{\rho, T}^{\prime}-\sum_{m=0}^{\rho-1}\left(\begin{array}{c}
\rho-1 \\
m-1
\end{array}\right) \mu_{\rho-m, T}^{\prime} \kappa_{m, T} .
$$

\subsection{Residual life and reversed residual life functions}

The $\hbar^{\text {th }}$ moment of the residual life

the $\hbar^{\text {th }}$ moment of the residual life of $T$ is given by

$$
v_{\hbar, T}(\tau)=E\left[\left.(T-\tau)^{\hbar}\right|_{T>\tau, \hbar=1,2, \ldots}\right]
$$

$$
v_{\hbar, T}(\tau)=\frac{1}{1-F(\tau)} \int_{\tau}^{\infty}(T-\tau)^{\hbar} d F(T) .
$$

Therefore, 
where

$$
v_{\hbar, T}(\tau)=\left.\frac{1}{1-F(\tau)} \sum_{s=0}^{\infty} \alpha^{\hbar} \vartheta_{s}^{(\hbar, \beta) *} \Gamma\left(1-\frac{\hbar}{\beta},(1+s)(a / \tau)^{\beta}\right)\right|_{\beta>\hbar},
$$

and

$$
\vartheta_{s}^{(\hbar, \beta) *}=\vartheta_{s} \sum_{\rho=0}^{\hbar}\left(\begin{array}{l}
\hbar \\
\rho
\end{array}\right)(-\tau)^{\hbar},\left.\Gamma(\nu, \rho)\right|_{\rho>0}=\int_{\rho}^{\infty} t^{\nu-1} \exp (-t) d t
$$

The $\hbar^{\text {th }}$ moment of the reversed residual life, say

$$
\Gamma(v, \rho)=\Gamma(v)-\gamma(v, \rho)
$$

uniquely determines $F(T)$. We obtain

$$
V_{\hbar, T}(\tau)=E\left[\left.(\tau-T)^{\hbar}\right|_{T \leq \tau, \tau>0} \text { and } \hbar=1,2, \ldots\right]
$$

$$
V_{\hbar, T}(\tau)=\frac{1}{F(\tau)} \int_{0}^{\tau}(\tau-T)^{\hbar} d F(t)
$$

Then, the $\hbar^{\text {th }}$ moment of the reversed residual life of $T$ becomes

where

$$
V_{\hbar, T}(\tau)=\left.\frac{1}{F(\tau)} \sum_{s=0}^{\infty} \alpha^{\hbar} \vartheta_{s}^{(\hbar, \beta) * *} \gamma\left(1-\frac{\hbar}{\beta},(1+s)(a / \tau)^{\beta}\right)\right|_{\beta>\hbar},
$$

$$
\vartheta_{s}^{(\hbar, \beta) * *}=\vartheta_{s} \sum_{\rho=0}^{\hbar}(-1)^{\rho}\left(\begin{array}{l}
\hbar \\
\rho
\end{array}\right) \tau^{\hbar-\rho} .
$$

\section{The maximum likelihood estimation (MLE) method}

Let $T_{1}, T_{2}, \ldots, T_{\hbar}$ be a random sample from size $\hbar$ from the OB-Fr distribution with parameters $a, b, \alpha$ and $\beta$. For determining the MLE of $\underline{P}$, we have the log-likelihood function

$$
\begin{aligned}
& \ell=\ell(P)=\hbar \log \left(a b \beta \alpha^{\beta}\right)-(\beta+1) \sum_{i=1}^{\hbar} \log \left(t_{i}\right)-a b \sum_{i=1}^{\hbar} \alpha^{\beta} t^{-\beta} \\
& +2 \sum_{i=1}^{\hbar} \log \left(\exp \left[-a b \alpha^{\beta} t^{-\beta}\right]+\left\{1-\exp \left[-b \alpha^{\beta} t^{-\beta}\right]\right\}^{a}\right)+(a-1) \sum_{i=1}^{\hbar} \log \left\{1-\exp \left[-b \alpha^{\beta} t^{-\beta}\right]\right\} .
\end{aligned}
$$

The components of the score vector is available if needed. Setting $U_{a}=U_{b}=U_{\alpha}=U_{\beta}=0$ and solving them simultaneously yields the MLEs. To solve these equations, it is usually more convenient to use nonlinear optimization methods such as the quasi-Newton algorithm to numerically maximize $\ell$. For interval estimation of the parameters, we obtain the $3 \times 3$ observed information matrix

$$
\mathbf{J}(P)=\left.\left\{\partial^{2} \ell / \partial r \partial s\right\}\right|_{(r, s=a, b, \alpha, \beta)}
$$

\section{Graphical assessment}

Graphically and using the biases and mean squared errors ( $M S E s$ ), we can perform the simulation experiments to assess the finite sample behavior of the MLEs. The assessment was based on the following algorithm:

$i$. Generate $N=1000$ samples of size $\left.n\right|_{(n=50,100, \ldots, 1500)}$ from the OB-Fr distribution using (7).

ii. $\quad$ Compute the MLEs for the $N=1000$ samples.

iii. $\quad$ Compute the standard errors (SEs) of the MLEs for the 1000 samples.

iv. Compute the biases and mean squared errors given for $\underline{P}=a, b, \alpha, \beta$. We repeated these steps for $\left.n\right|_{(n=50,100, \ldots, 1500)}$ with $a=b=\alpha=\beta=1$, so computing biases ( $\operatorname{Bias}_{\underline{P}}(n)$ ), mean squared errors ( $\operatorname{MSES})\left(\operatorname{MSE}_{h}(n)\right)$ for $\underline{P}=a, b, \alpha, \beta$ and $\left.n\right|_{(n=50,100, \ldots, 1500)}$. 


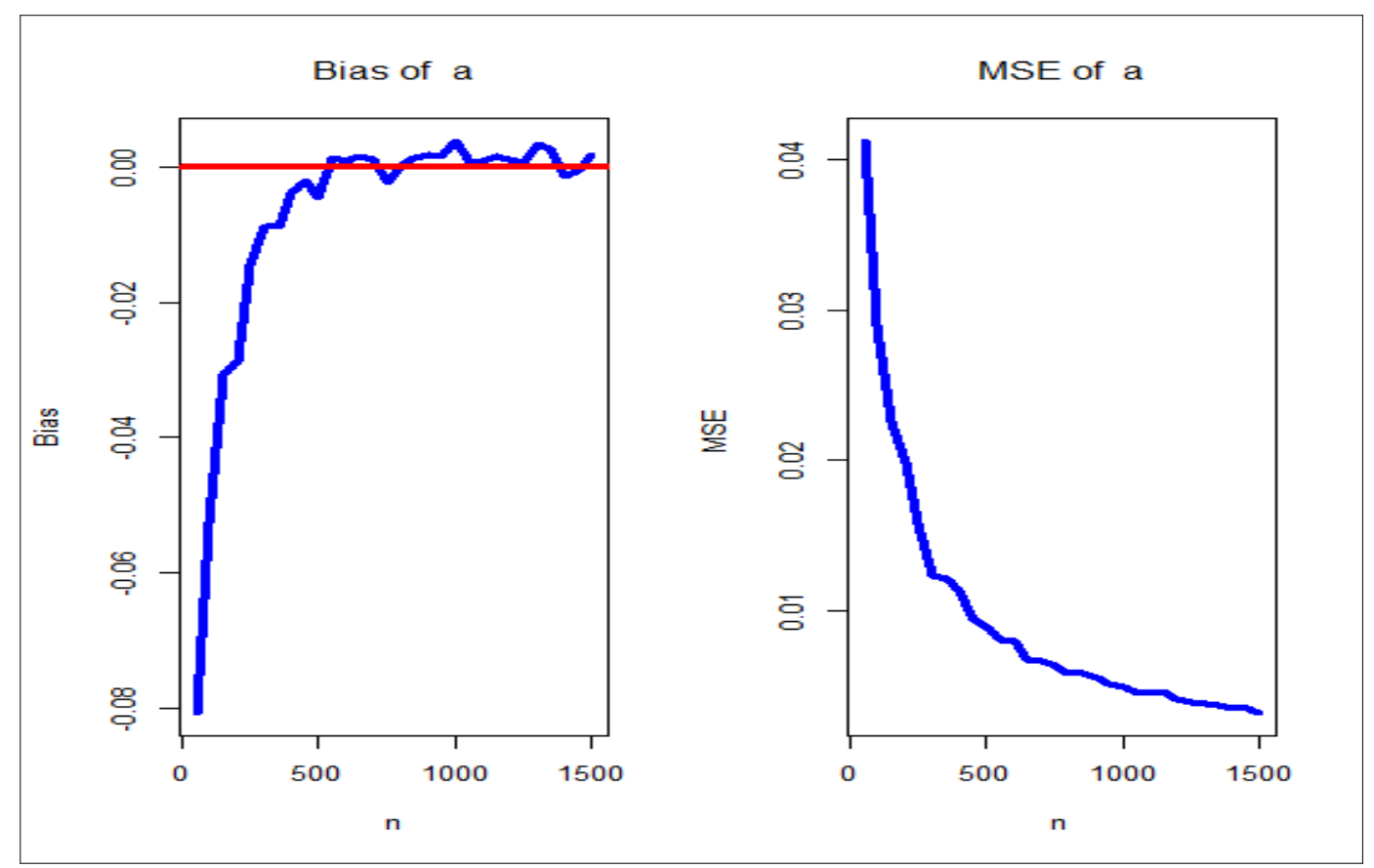

Figure 1: biases and mean squared errors for the parameter $a$.
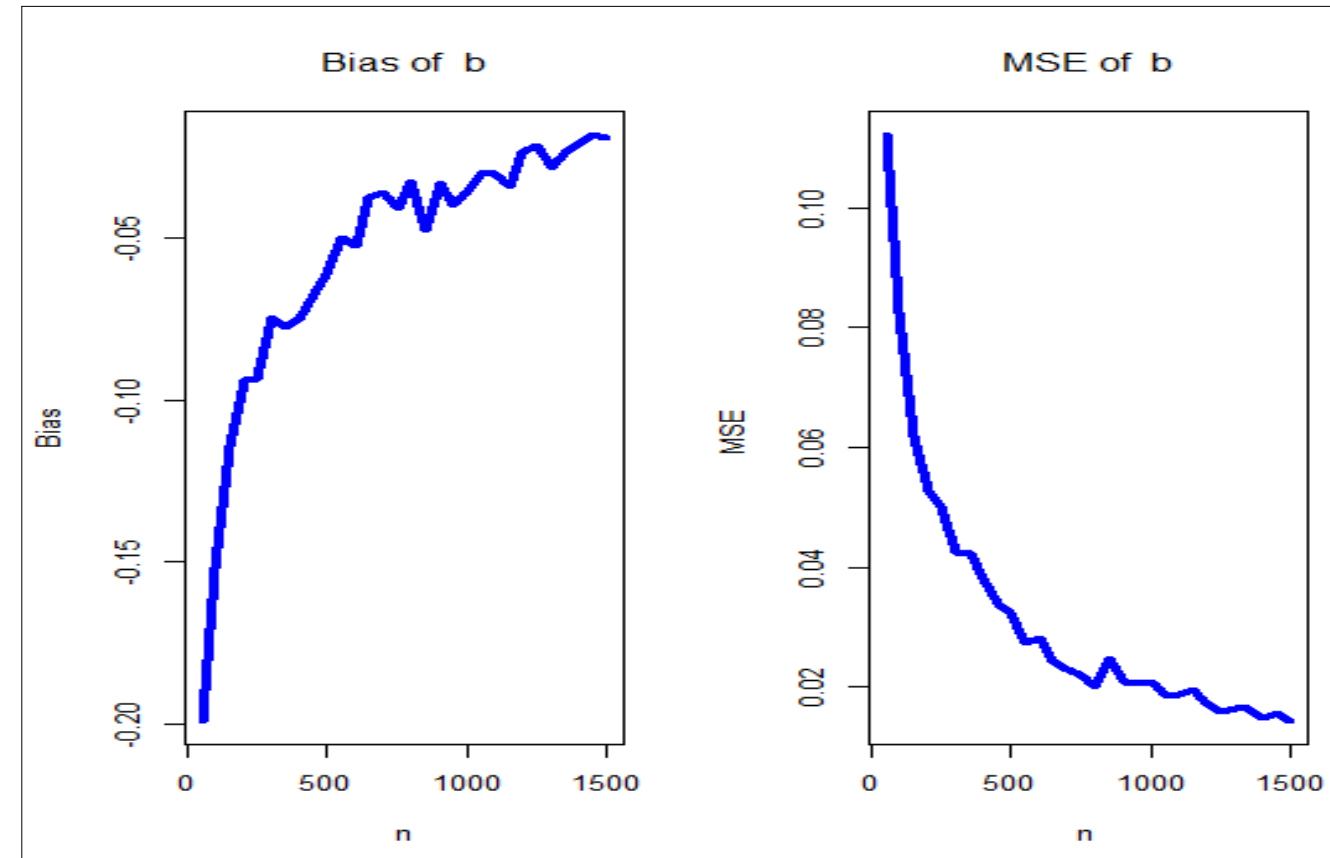

Figure 2: biases and mean squared errors for the parameter $b$. 


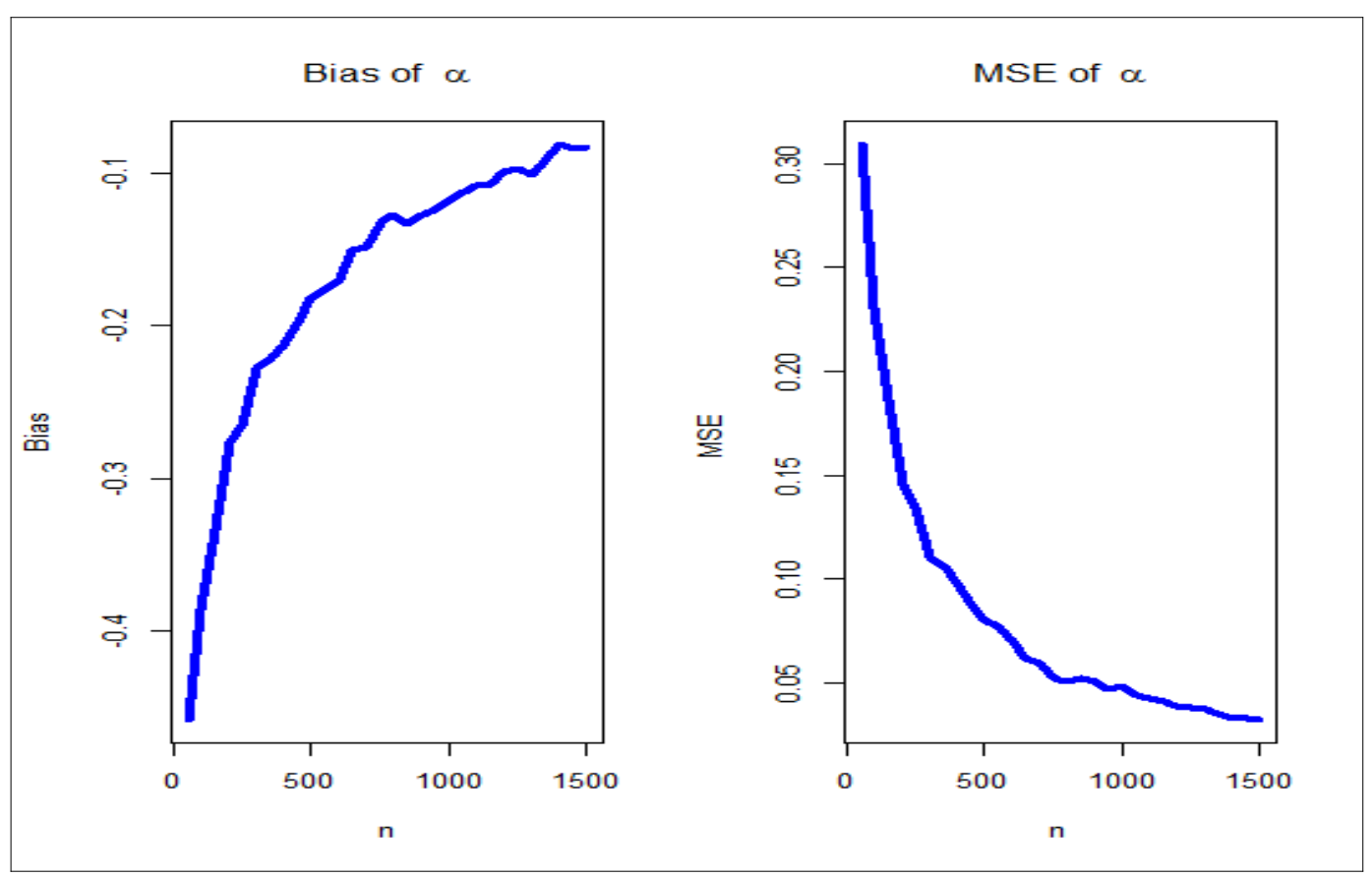

Figure 3: biases and mean squared errors for the parameter $(-)$

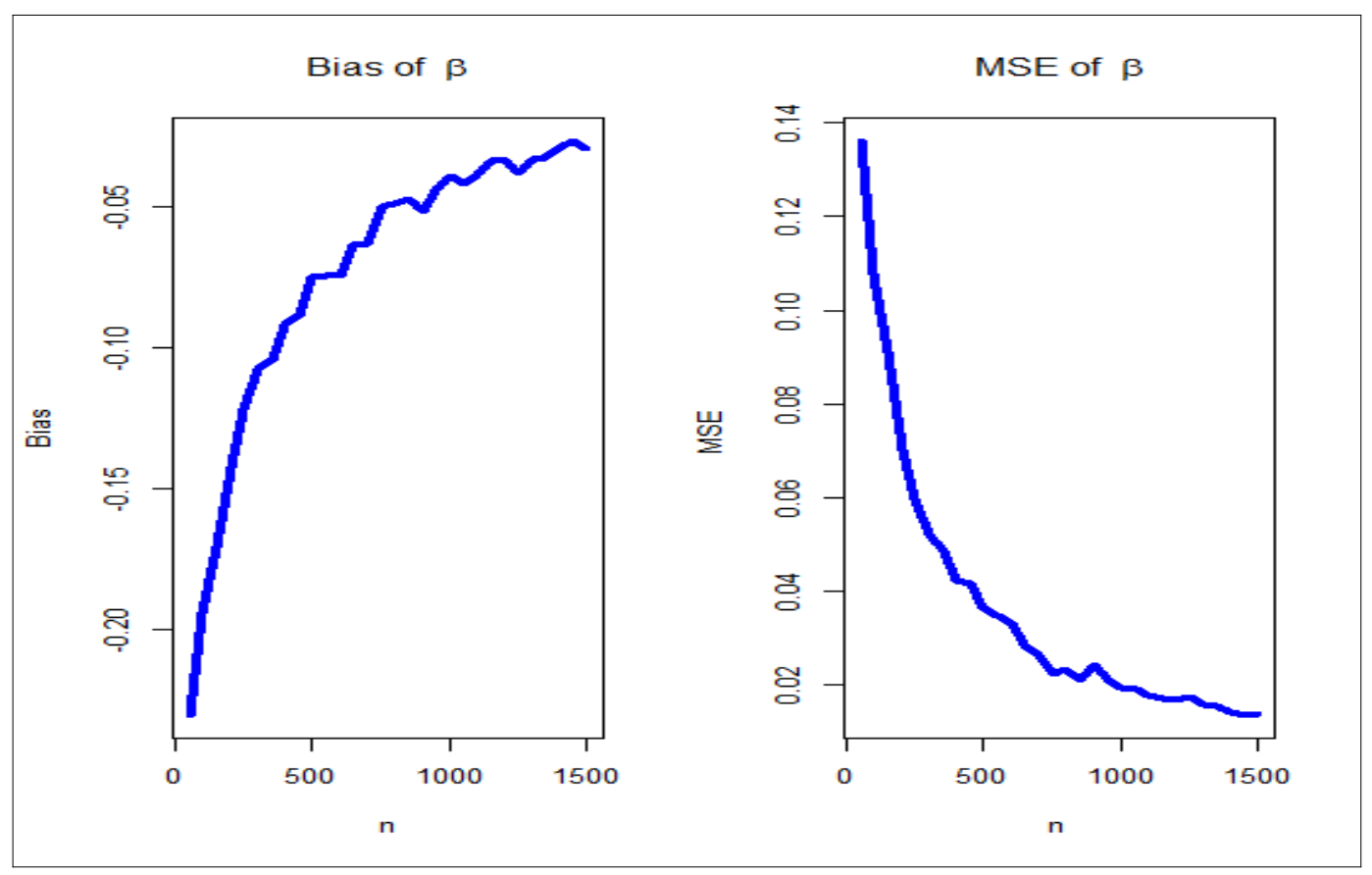

Figure 4: biases and mean squared errors for the parameter $\Theta$

Figures 1, 2, 3 and 4 gives the biases (left panel) and MSEs (right panel) for the parameters $\alpha, \beta, a$ and $b$ respectively. These Figures (left panels) show how the four biases vary with respect to $n$ and also the right panels 
show how the four MSEs vary with respect to $n$. The broken line in Figure 1 corresponds to the biases being 0 . From Figures 1-4, the biases for each parameter are generally negative and decrease to zero as $n \rightarrow \infty$, the MSEs for each parameter decrease to zero as $n \rightarrow \infty$.

\section{Modeling uncensored real data for comparing the competitive models}

For illustrating the wide applicability of the new OB-Fr model, we consider the Cramér-Von Mises (CVM) statistic, the Anderson-Darling (A-D) statistic, the Kolmogorov-Smirnov (KS) statistic and its corresponding p-value $(P) v)$. Table 3 below gives the competitive models.

Table 3: The competitive models.

\begin{tabular}{cc}
\hline Competitive models (Abbreviation) & Author(s) \\
\hline Fréchet (Fr) & Fréchet (1927). \\
Exponentiated-Fr (E-Fr) & Nadarajah and Kotz (2003). \\
Beta-Fr (B-Fr) & Barreto-Souza et al. (2011). \\
Marshal-Olkin-Fr (MO-Fr) & Krishna et al. (2013). \\
Transmuted-Fr (T-Fr) & Mahmoud and Mandouh (2013). \\
Kumaraswamy-Fr (Kum-Fr) & Mead and Abd-Eltawab (2014). \\
McDonald-Fr (Mc-Fr) & Shahbaz et al. (2016). \\
odd log-logistic-IR (OLL-IR) & Yousof et. al (2018a). \\
odd log-logistic-EFr (OLL-EF) & - \\
odd log-logistic-EFr (OLL-EIR) & - \\
Generalized odd log-logistic-IR(GOLL-IR) & - \\
\hline
\end{tabular}

\subsection{Stress data}

The $1 s \tau$ data set is an uncensored data set consisting of 100 observations on breaking stress of carbon fibers (in Gba) given by Nichols and Padgett (2006) and these data are used by Mahmoud and Mandouh (2013) to fit the transmuted Fr distribution. Figure 5 gives in its left panel the total time test (TTT) plot (see Aarset (1987) for more details) for data set I along with the box plot for discovering the EVs. It indicates that the empirical HRF of data sets I is "increasing HRF" and we have six EVs. The statistics CVM, A-D , K-S and $\mathbf{P} v$ for all fitted models are presented in Table 4. The MLEs and corresponding standard errors (SEs) are given in Table 5. From Table 4, the OB-Fr model gives the lowest values the $\mathrm{CVM}=\mathbf{0 . 0 6 6 4}, \mathrm{A}-\mathrm{D}=\mathbf{0 . 4 7 0 6}, \mathrm{K}-\mathrm{S}=\mathbf{0 . 0 6 3 0}$ and $\mathbf{P} \boldsymbol{v}=\mathbf{0 . 8 2 2}$ as compared to further Fr models. Therefore, the OB-Fr can be chosen as the best model. Figure 6 gives the estimated (E-PDF) versus the estimated CDF (E-CDF). Figure 6 gives the Probability-Probability (P-P) plot and estimated HRF (E-HRF) for data set I. From Figures 6 and 7, we note that the new OB-Fr model provides adequate fits to the empirical functions.

Table 4: CVM, A-D, K-S and P_\{v $\}$ for data set I.

\begin{tabular}{ccccc}
\hline \multirow{2}{*}{ Model } & \multicolumn{4}{c}{ Goodness of fit criteria } \\
\cline { 2 - 5 } & CVM & A-D & K-S & P $v$ \\
\hline OB-Fr & 0.0664 & 0.4706 & 0.0630 & 0.822 \\
OLLE-Fr & 0.1203 & 0.9639 & 0.5561 & $2.2 \times 10^{-16}$ \\
OLLE-IR & 0.1553 & 1.21197 & 0.65497 & $2.2 \times 10^{-16}$ \\
OLL-IR & 0.1553 & 1.21201 & 0.6550 & $2.2 \times 10^{-16}$ \\
Fr & 0.1090 & 0.7657 & 0.0874 & 0.4282 \\
Kum-Fr & 0.0812 & 0.6217 & 0.0759 & 0.6118 \\
Exp-Fr & 0.1091 & 0.7658 & 0.0874 & 0.4287 \\
Beta-Fr & 0.0809 & 0.6207 & 0.0757 & 0.6147 \\
T-Fr & 0.0871 & 0.6209 & 0.0782 & 0.5734 \\
MO-Fr & 0.0886 & 0.6142 & 0.0763 & 0.5168 \\
Mc-Fr & 0.1333 & 1.0608 & 0.0807 & 0.5332 \\
OLLE-IR & 0.1553 & 1.21197 & 0.65497 & $2.2 \times 10^{-16}$ \\
OLL-IR & 0.1553 & 1.21201 & 0.6550 & $2.2 \times 10^{-16}$ \\
\hline
\end{tabular}


Table 5: MLEs and their standard errors (in parentheses) for data set I.

\begin{tabular}{cccccc}
\hline \multirow{2}{*}{ Model } & \multicolumn{5}{c}{ Estimates } \\
\cline { 2 - 6 } OB-Fr & $\mathrm{a}$ & $\mathrm{b}$ & $\mathrm{c}$ & $\alpha$ & $\beta$ \\
& 5.1954 & 0.5990 & & 1.0404 & 1.232 \\
OLLExp-Fr & $(0.000)$ & $(0.000)$ & & $(0.000)$ & $(0.000)$ \\
& 0.1351 & & 3.7216 & 0.9296 & 21.319 \\
OLLE-IR & $(0.011)$ & & $(0.0034)$ & $(0.0033)$ & $(0.0034)$ \\
& 0.4946 & & 0.067 & 1.74262 & \\
OLL-IR & $(0.04135)$ & & $(0.7195)$ & $(9.3007)$ & \\
& 0.49459 & & & 0.45242 & \\
Fr & 0.04135 & & & 0.03869 & \\
& & & & 1.3968 & 4.3724 \\
Kum-Fr & & 0.8489 & 1.6239 & $(0.0336)$ & $(0.3278)$ \\
& & $(16.083)$ & $(0.6979)$ & $(9.049)$ & 3.4208 \\
Exp-Fr & & 0.9395 & & 1.4169 & $0.7635)$ \\
& & $(3.543)$ & & $(2.568)$ & $(0.3278)$ \\
Beta-Fr & & 0.7346 & 1.5830 & 1.6684 & 3.5112 \\
& & $(1.5290)$ & $(0.7132)$ & $(0.7662)$ & $(0.9683)$ \\
T-Fr & -0.7166 & & & 1.2656 & 4.7121 \\
& $(0.2616)$ & & & $(0.0579)$ & $(0.3657)$ \\
MO-Fr & & 0.0033 & & 6.2296 & 1.2419 \\
& & $(0.0009)$ & & $(1.0134)$ & $(0.1181)$ \\
Mc-Fr & 0.8503 & 44.423 & 19.859 & 0.0203 & 46.974 \\
& $(0.1353)$ & $(25.100)$ & $(6.706)$ & $(0.0060)$ & $(21.871)$ \\
\hline
\end{tabular}
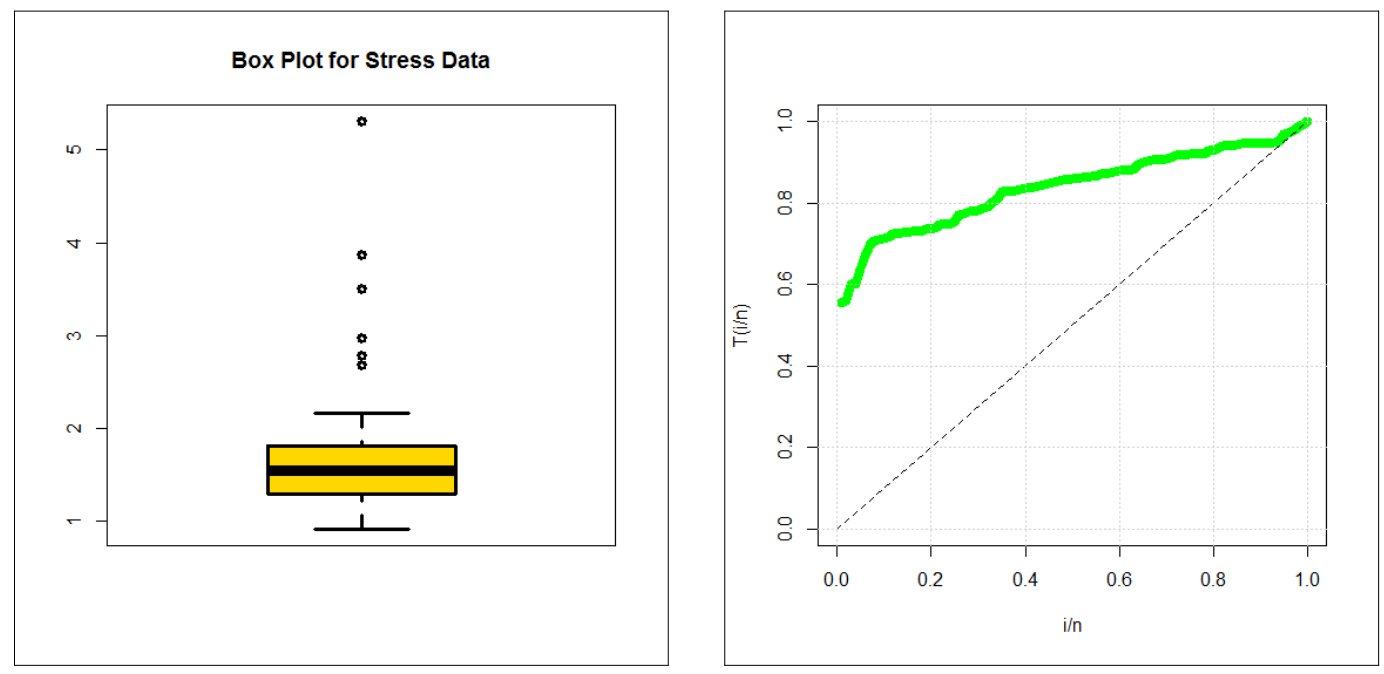

Figure 5: Box plot (left panel) and TTT plot (right panel) for data set I. 

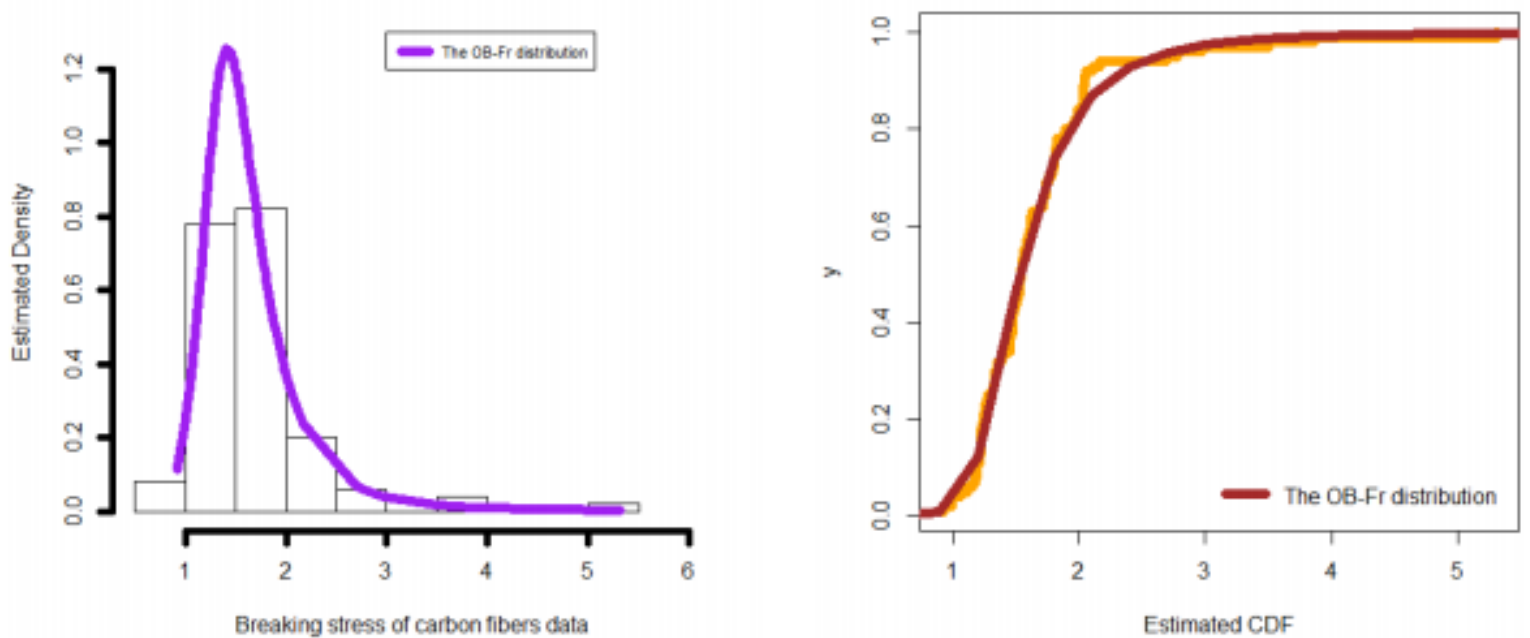

Figure 6: Estimated density, estimated CDF for data set I.
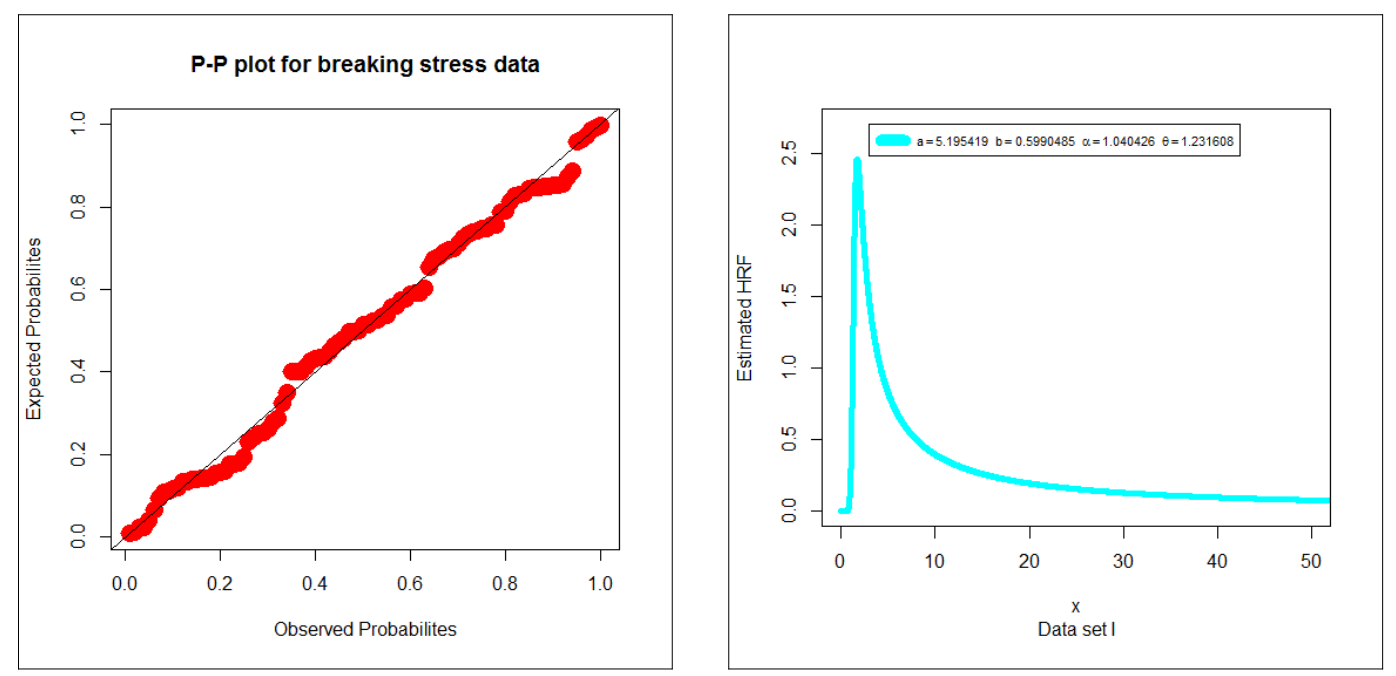

Figure 7: P-P plot and estimated HRF for data set $\mathbf{I}$.

\subsection{Glass fibers data}

The 2 nd data set is generated data to simulate the strengths of glass fibers which was given by Smith and Naylor (1987). Figure 8 gives in its left panel the TTT for data set II along with its corresponding box plot for discovering the EVs. It indicates that the empirical HRF of data sets II is "increasing HRF" and we have four EVs. The statistics CVM, A-D , K-S and $\mathbf{P} v$ for all fitted models are presented in Table 6. The MLEs and corresponding SEs are given in Table 7. From Table 6, the OB-Fr model gives the lowest values the $\mathrm{CVM}=\mathbf{0 . 0 5 4 4 7}, \mathrm{A}-\mathrm{D}=\mathbf{0 . 3 8 5 8}, \mathrm{K}-\mathrm{S}=\mathbf{0 . 0 7 9 7}$ and $\mathbf{P} v=\mathbf{0 . 8 8 2 7 0}$ as compared to further Fr models. Therefore, the OB-Fr can be chosen as the best model. Figure 9 gives the E-PDF versus the E-CDF. Figure 10 gives the P-P plot and E-HRF for data set II. based on Figures 9 and 10, we note that the new OB-Fr model provides adequate fits to the empirical functions.

Table 6: CVM, A-D, K-S and P_ $\{\mathrm{v}\}$ for data set II.

\begin{tabular}{ccccc}
\hline Model & \multicolumn{4}{c}{ Goodness of fit criteria } \\
\cline { 2 - 5 } & CVM & A-D & K-S & P $v$ \\
\hline OB-Fr & 0.05447 & 0.3858 & 0.0797 & 0.88270 \\
Fr & 0.0707 & 0.5332 & 0.0772 & 0.8185 \\
Exp-Fr & 0.0707 & 0.5332 & 0.0772 & 0.8187
\end{tabular}




\begin{tabular}{ccccc} 
OLLE-Fr & 0.10487 & 0.8325 & 0.55196 & $6.7 \times 10^{-16}$ \\
OLLE-IR & 0.1502 & 1.14697 & 0.67949 & $6.7 \times 10^{-16}$ \\
OLL-IR & 0.15021 & 1.14697 & 0.67951 & $6.7 \times 10^{-16}$ \\
Kum-Fr & 0.0634 & 0.4981 & 0.0715 & 0.8810 \\
MO-Fr & 0.0629 & 0.4902 & 0.0813 & 0.7685 \\
Beta-Fr & 0.0640 & 0.5008 & 0.0716 & 0.8804 \\
T-Fr & 0.0655 & 0.4939 & 0.0735 & 0.8470 \\
Mc-Fr & 0.1161 & 0.9193 & 0.0831 & 0.7455 \\
\hline
\end{tabular}

Table 7: MLEs and their standard errors (in parentheses) for data set II.

\begin{tabular}{cccccc}
\hline Model & \multicolumn{5}{c}{ Estimates } \\
\cline { 2 - 6 } OB-Fr & $\mathrm{a}$ & $\mathrm{b}$ & $\mathrm{c}$ & $\alpha$ & $\beta$ \\
& $(0.4757$ & 0.4976 & & 1.0467 & 1.338 \\
OLLExp-Fr & 0.1449 & $(0.000)$ & & $(0.000)$ & $(0.000)$ \\
& $(0.0129)$ & & 0.00879 & 1.2997 & 24.878 \\
OLLE-IR & 0.5025 & & $(0.000)$ & $(0.000)$ & $(0.000)$ \\
& $(0.0529)$ & & $(1.0716$ & 1.7048 & \\
OLL-IR & 0.50251 & & & $0.4559)$ & \\
& $(0.0529)$ & & & $(0.0486)$ & \\
Fr & & & & 1.4108 & 5.4377 \\
& & & & $(0.0344)$ & $(0.5192)$ \\
Kum-Fr & & 0.2855 & 1.2824 & 1.9142 & 4.7731 \\
& & $(9.1338)$ & $(0.6388)$ & $(12.836)$ & $(1.3134)$ \\
Exp-Fr & & 0.9059 & & 1.4367 & 5.4379 \\
& & $(2.764)$ & & $(4.324)$ & $(0.5193)$ \\
B-Fr & & 1.2996 & 1.2649 & 1.3945 & 4.7927 \\
& & $(4.4378)$ & $(0.6640)$ & $(0.9304)$ & $(1.4641)$ \\
T-Fr & 0.7778 & & & 1.5491 & 4.3139 \\
& $(0.2477)$ & & & $(0.0655)$ & $(0.5849)$ \\
MO-Fr & & 0.0023 & & 5.2383 & 1.4537 \\
& & $(0.0004)$ & & $(0.8209)$ & $(0.1650)$ \\
Mc-Fr & & 56.227 & 14.953 & 0.0073 & 29.104 \\
& & $(30.539)$ & $(4.733)$ & $(0.0013)$ & $(11.304)$ \\
\hline
\end{tabular}



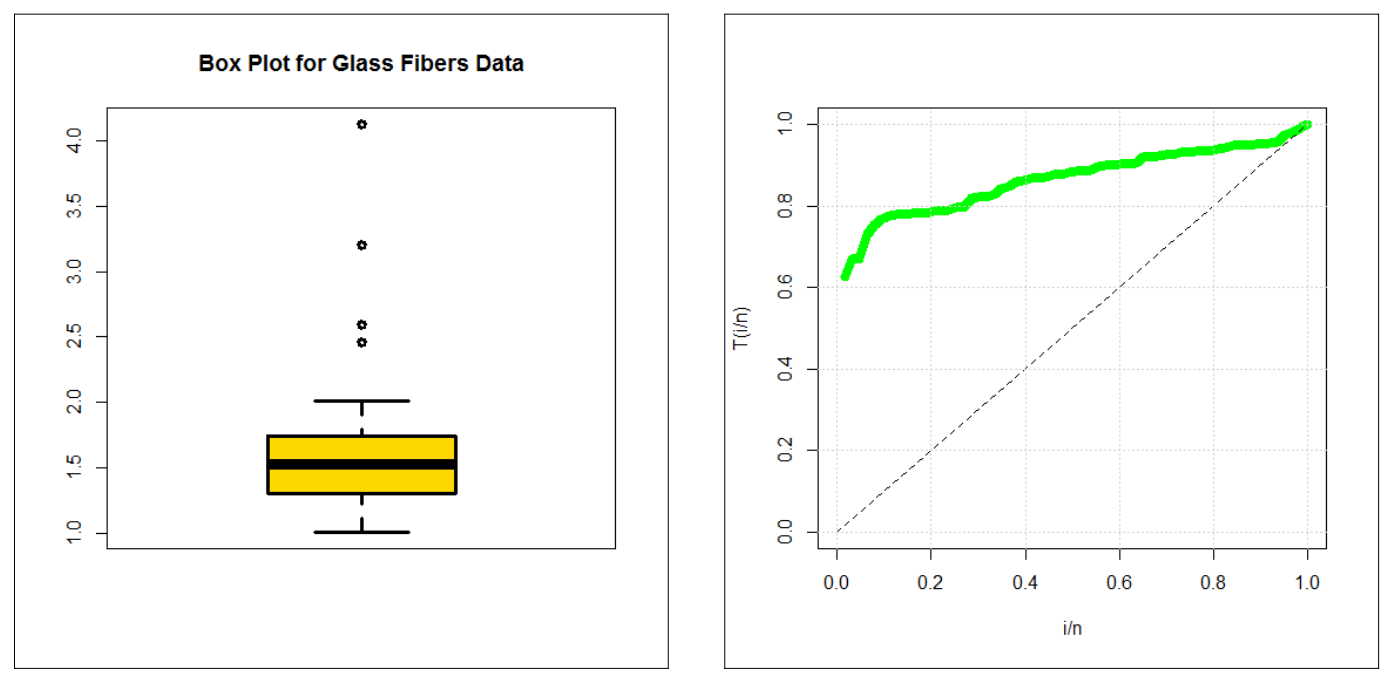

Figure 8: Box plot (left panel) and TTT plot (right panel) for data set II.
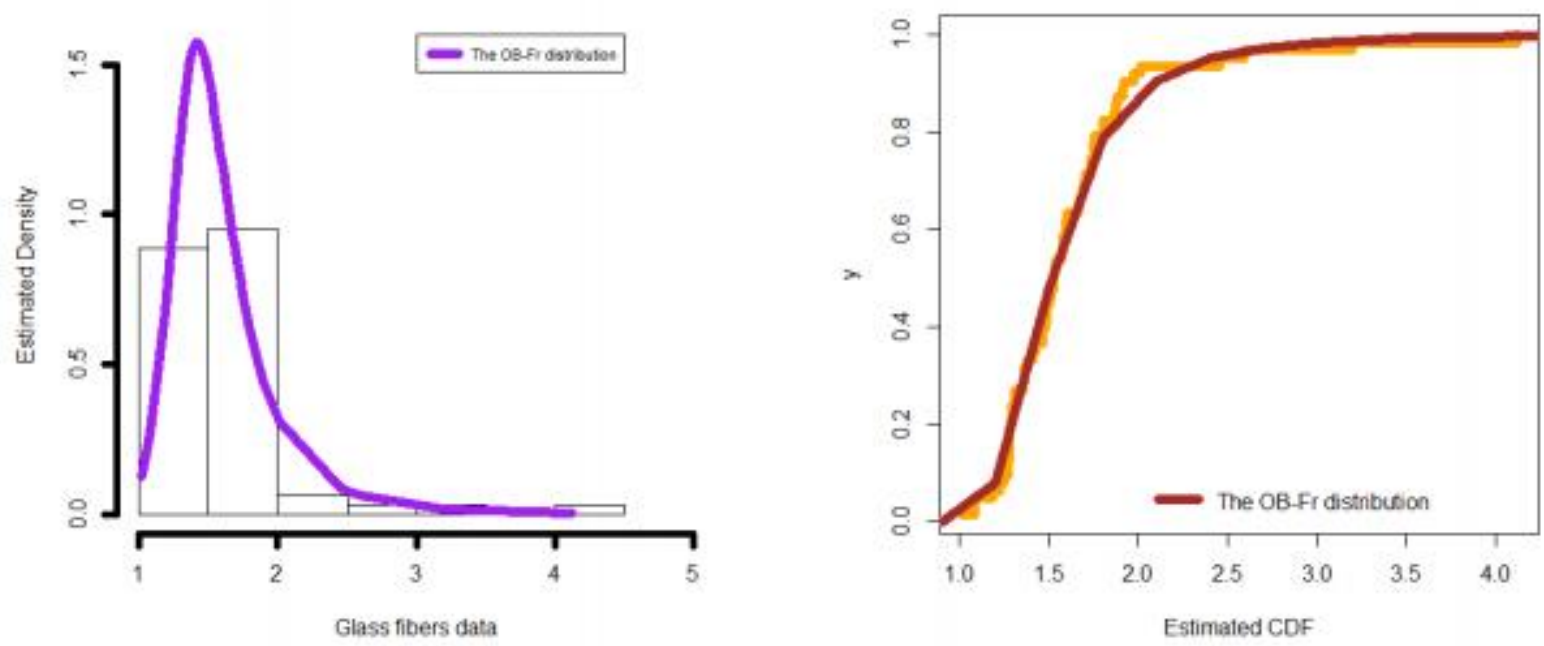

Figure 9: Estimated density, estimated CDF, P-P plot and estimated HRF for data set II. 

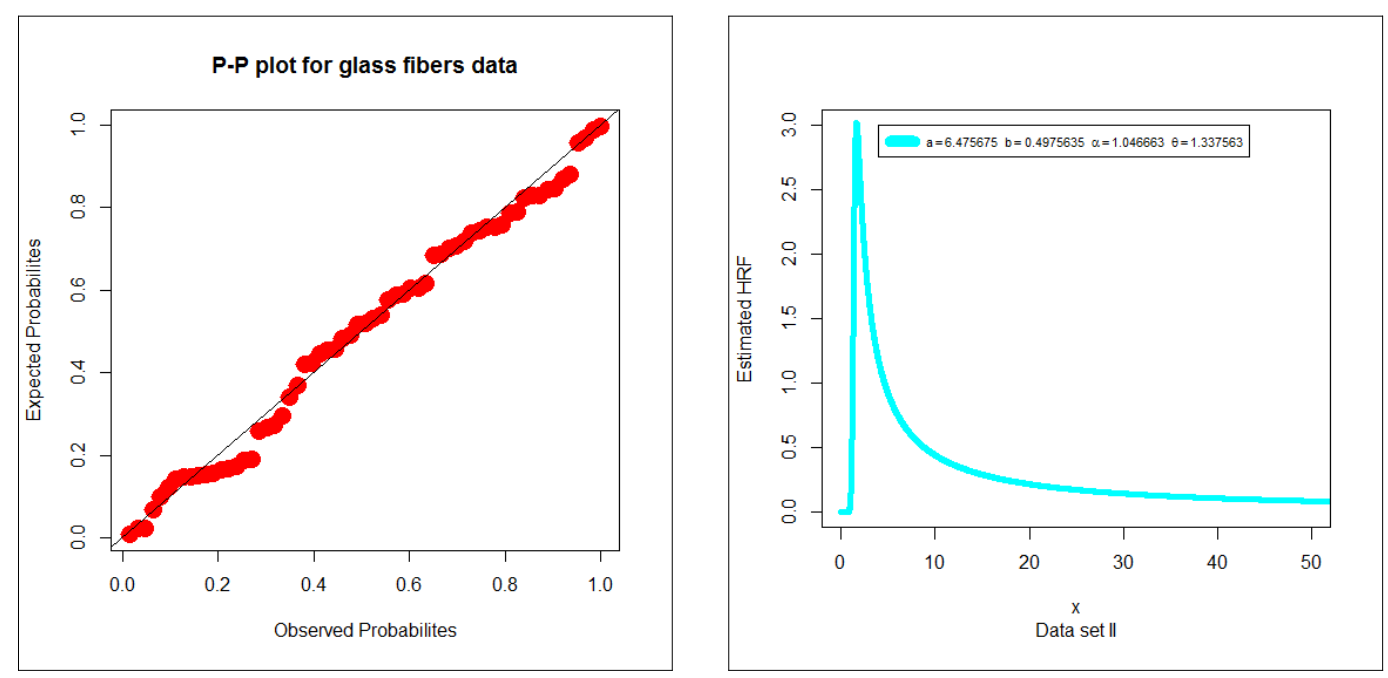

Figure 10: P-P plot and estimated HRF for data set II.

\subsection{Relief time data}

The $3 \mathrm{rd}$ data set (wingo data) represents a complete sample from a clinical trial describe a relief time (in hours) for 50 arthritic patients. Figure 11 gives in its left panel the TTT for data set III along with its corresponding box plot for discovering the EVs. Based on Figure 11, the empirical HRF of data sets III is "increasing HRF" and we have no EVs. The statistics CVM, A-D , K-S and $\mathbf{P} v$ for all fitted models are presented in Table 6. The MLEs and corresponding SEs are given in Table 7. From Table 6, the OB-Fr model gives the lowest values the CVM=0.04903, A-D=0.42081, $\mathrm{K}-\mathrm{S}=\mathbf{0 . 0 9 1 2 4}$ and $\mathbf{P} v=\mathbf{0 . 7 9 9 4}$. Therefore, the OB-Fr may be chosen as the best model. Figure 12 gives the E-PDF versus the E-CDF. Figure 13 gives the P-P plot and E-HRF for data set III. based on Figures 12 and 13, we note that the new OB-Fr model provides adequate fits to the empirical functions.

Table 8: CVM, A-D, K-S and P_\{v $\}$ for data set III.

\begin{tabular}{ccccc}
\hline \multirow{2}{*}{ Model } & \multicolumn{4}{c}{ Goodness of fit criteria } \\
\cline { 2 - 5 } & CVM & A-D & K-S & P $v$ \\
\hline OB-Fr & 0.0490 & 0.42081 & 0.09124 & 0.7994 \\
GOLLIR & 0.1955 & 1.3498 & 0.11008 & 0.5797 \\
OLLExp-Fr & 0.1577 & 1.09876 & 0.53498 & $7.4 \times 10^{-13}$ \\
Fr & 0.3233 & 2.0301 & 0.1506 & 0.2066 \\
Exp-Fr & 0.3233 & 2.0301 & 0.1506 & 0.2064 \\
Beta-Fr & 0.3611 & 2.5131 & 0.1433 & 0.3601 \\
T-Fr & 0.2823 & 1.8152 & 0.1370 & 0.3045 \\
\hline
\end{tabular}

Table 7: MLEs and their standard errors (in parentheses) for data set II.

\begin{tabular}{cccccc}
\hline \multirow{2}{*}{ Model } & \multicolumn{5}{c}{ Estimates } \\
\cline { 2 - 6 } & $\mathrm{a}$ & $\mathrm{b}$ & $\mathrm{c}$ & $\alpha$ & $\beta$ \\
\hline OB-Fr & 17.791 & 6.9955 & & 0.12686 & 0.17843 \\
& $(0.0001)$ & $(4.0354)$ & & $(0.000)$ & $(0.000)$ \\
GOLLIR & 1.961 & 0.111 & & 1.4123 & \\
& $(0.234)$ & $(0.000)$ & & $(0.000)$ & \\
OLLExp-Fr & 0.0669 & & 0.00459 & 0.3558 & 32.561 \\
& $(0.0076)$ & & $(0.0028)$ & $(0.0047)$ & $(0.006)$ \\
Fr & & & & 0.4859 & 3.2078 \\
& & & & $(0.0227)$ & $(0.3263)$
\end{tabular}



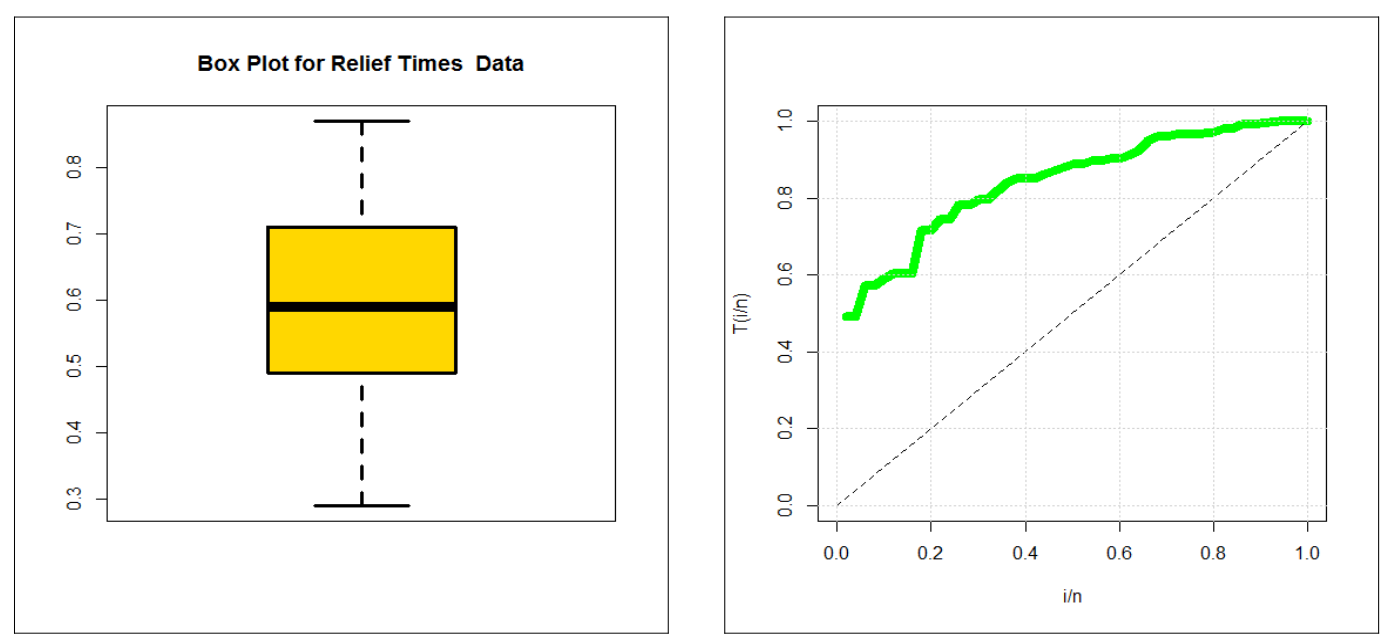

Figure 11: Box plot (left panel) and TTT plot (right panel) for data set III.
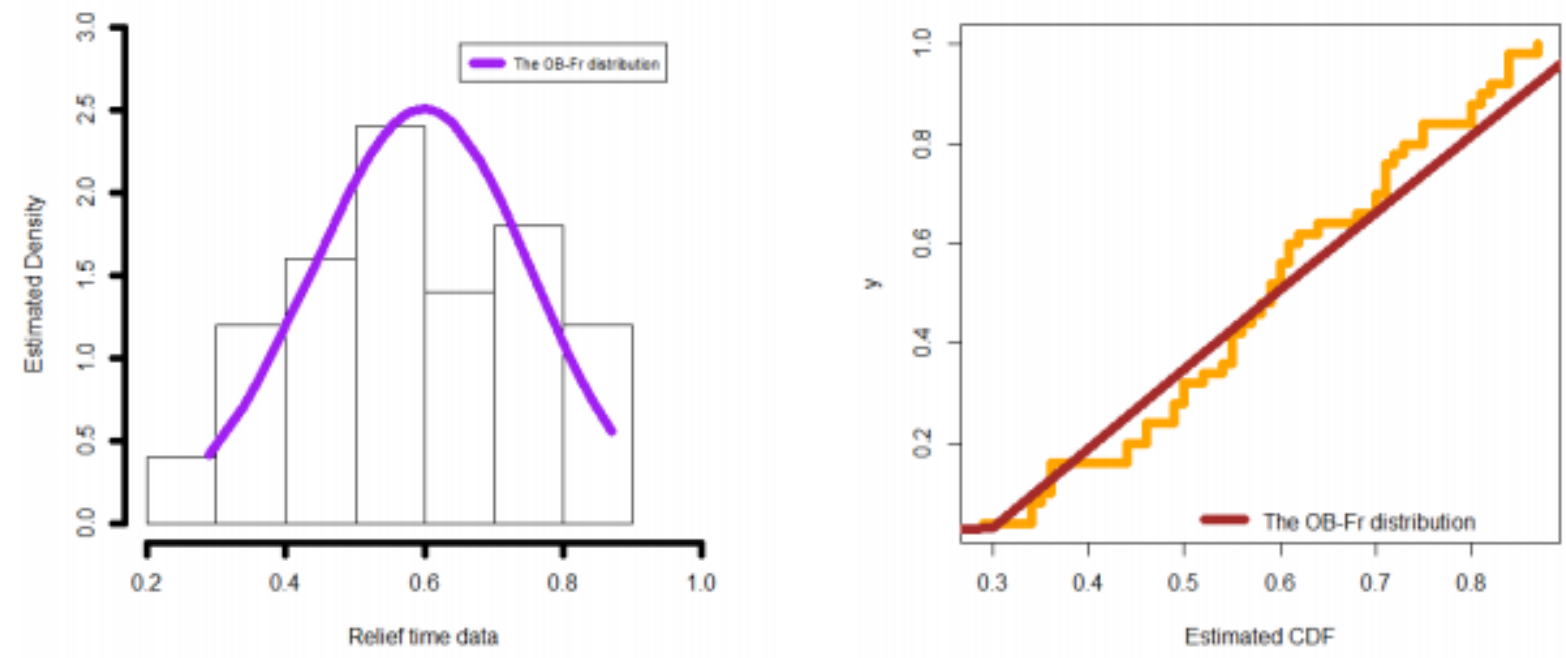

Figure 12: Estimated density, estimated CDF, P-P plot and estimated HRF for data set III. 

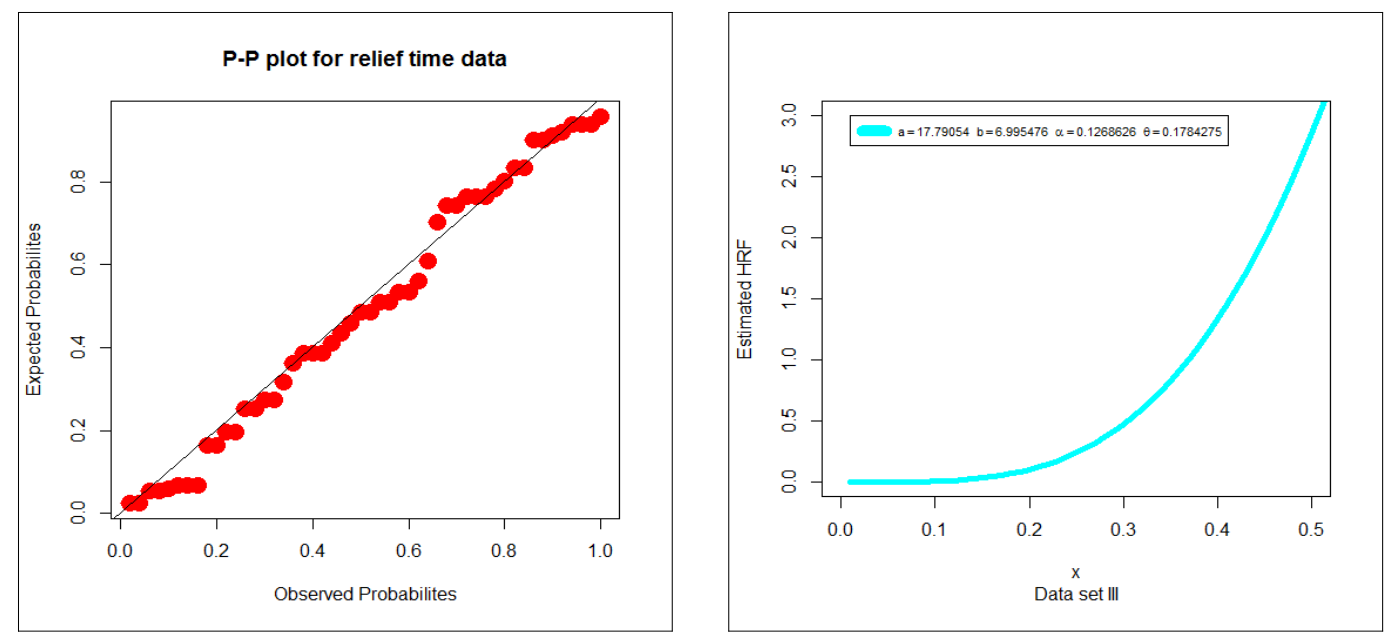

Figure 13: P-P plot and estimated HRF for data set III.

\section{Concluding remarks}

In this article and based on Alizadeh et al. (2016), we expanded the extreme value theory with proposing and studying a new version of the Fréchet model called the generalized Odd-Burr generalized Fréchet model. A straightforward types copula based on Farlie Gumbel Morgenstern family, modified Farlie Gumbel Morgenstern family, Clayton Copula and Renyi's entropy are employed for proposing many bivariate and multivariate type extensions. Some of its mathematical properties such as ordinary moments, incomplete moments, moment generating function, cumulant generating function, residual life and reversed residual life functions are derived. A numerical analysis for some measures including the Mean, variance, skewness, kurtosis and Index of dispersion for the new model are presented with details. The maximum likelihood estimation method is employed to estimate the model parameters. Graphically and using the biases and mean squared errors, we performed the simulation experiments to assess of the finite sample behavior of the maximum likelihood estimations. Three applications are presented for measuring the flexibility and the importance of the new model are presented and also used for comparing the competitive distributions under the uncensored scheme.

As a future potential work, we can modify many new useful goodness-of-fit tests for the right censored distributional validation such as the Nikulin-Rao-Robson goodness-of-fit test statistic, modified Nikulin-Rao-Robson goodness-offit test statistic, Bagdonavicius-Nikulin goodness-of-fit test statistic and also modified Bagdonavicius-Nikulin goodness-of-fit test statistic to the new BuXENH model as performed by Ibrahim et al. (2019), Goual et al. (2019, 2020), Mansour et al. (2020a,b,c,d), Yadav et al. (2020) and Goual and Yousof (2020), among others. However, many types of copulas can be used and applied for deriving many new bivariate models based on the new distribution (see Elgohari and Yousof (2020a), Mansour et al. (2020e, f) and El-Morshedy et al. (2021) for more details).

\section{References}

1. Aarset, M. V. (1987). How to identify a bathtub hazard rate. IEEE Transactions on Reliability, 36(1), 106-108.

2. Barreto-Souza, W. M., Cordeiro, G. M. and Simas, A. B. (2011). Some results for beta Fréchet distribution. Commun. Statist. Theory-Meth., 40, 798-811.

3. Al-Babtain, A. A. Elbatal, I. and Yousof, H. M. (2020). A new three parameter Fréchet model with mathematical properties and applications. Journal of Taibah University for Science, 14(1), 265-278.

4. Ali, M. M., Yousof, H. M. and Ibrahim, M. (2021a). A new version of the generalized Rayleigh distribution with copula, properties, applications and different methods of estimation. Optimal Decision Making in Operations Research \& Statistics: Methodologies and Applications, VOL 1, 1-25.

5. Ali, M. M., Ibrahim, M. and Yousof, H. M. (2021b). Expanding the Burr X model: properties, copula, real data modeling and different methods of estimation. Optimal Decision Making in Operations Research \& Statistics: Methodologies and Applications, VOL 1, 26-49.

6. Almazah, M.M.A., Almuqrin, M.A., Eliwa, M.S., El-Morshedy, M., Yousof, H.M. Modeling Extreme Values Utilizing an Asymmetric Probability Function. Symmetry 2021, 13, 1730. 
7. Chakraborty, S., Handique, L., Altun, E., \& Yousof, H. M. (2019). A New Statistical Model for Extreme Values: Properties and Applications. Int. J. Open Problems Compt. Math, 12(1).

8. Cordeiro, G. M., Alizadeh, M.M., Ozel, G., Hosseini, B., Ortega, E. M. M. and Altun, E. (2016). The generalized odd loglogistic family of distributions: properties, regression models and applications, Journal of Statistical Computation and Simulation, 87(5), 908-932.

9. Elgohari, H. and Yousof, H. M. (2020a). New Extension of Weibull Distribution: Copula, Mathematical Properties and Data Modeling. Statistics, Optimization \& Information Computing, 8(4), 972-993.

10. Elgohari, H. and Yousof, H. M. (2020b). New Extension of Weibull Distribution: Copula, Mathematical Properties and Data Modeling. Statistics, Optimization \& Information Computing, 8(4), 972-993.

11. Elgohari, H., Ibrahim, M. and Yousof, H. M. (2021). A New Probability Distribution for Modeling Failure and Service Times: Properties, Copulas and Various Estimation Methods. Statistics, Optimization \& Information Computing, 8(3), 555-586.

12. El-Morshedy, M., Alshammari, F. S., Hamed, Y. S., Eliwa, M. S., Yousof, H. M. (2021). A New Family of Continuous Probability Distributions. Entropy, 23, 194.

13. Elsayed, H. A. H. and Yousof, H. M. (2020). The generalized odd generalized exponential Fréchet model: univariate, bivariate and multivariate extensions with properties and applications to the univariate version. Pakistan Journal of Statistics and Operation Research, 529-544.

14. Fisher, R. A. and Tippett, L. H. C. (1928). Limiting forms of the frequency distribution of the largest or smallest member of a sample. In Mathematical Proceedings of the Cambridge Philosophical Society, pages 180--190. Cambridge Univ Press.

15. Fréchet, M. (1927). Sur la loi de probabilité de lécart maximum. Ann. de la Soc. polonaisede Math, 6, 93--116.

16. Ghosh, I. and Ray. S. (2016). Some alternative bivariate Kumaraswamy type distributions via copula with application in risk management. Journal of Statistical Theory and Practice 10, 693--706.

17. Gleaton, J. U. and Lynch, J.D. (2006). Properties of generalized loglogistic families of lifetime distributions. Journal of Probability and Statistical Science, 4, 51-64.

18. Goual, H., Yousof, H. M., \& Ali, M. M. (2019). Validation of the odd Lindley exponentiated exponential by a modified goodness of fit test with applications to censored and complete data. Pakistan Journal of Statistics and Operation Research, 15(3), 745-771.

19. Goual, H., \& Yousof, H. M. (2020). Validation of Burr XII inverse Rayleigh model via a modified chi-squared goodness-offit test. Journal of Applied Statistics, 47(3), 393-423.

20. Goual, H., Yousof, H. M., \& Ali, M. M. (2020). Lomax inverse Weibull model: properties, applications, and a modified Chisquared goodness-of-fit test for validation. Journal of Nonlinear Sciences \& Applications (JNSA), 13(6), 330-353.

21. Gumbel, E. J. (1961). Bivariate logistic distributions. Journal of the American Statistical Association, 56(294), 335-349.

22. Gumbel, E. J. (1960) Bivariate exponential distributions. Journ. Amer. Statist. Assoc., 55, 698-707.

23. Gupta, R. C. and Gupta, R. D. (2007). Proportional reversed hazard rate model and its applications. J. Statist Plan Inference.137, 3525--3536.

24. Gusmao, F. R. S., Ortega, E. M. M. and Cordeiro, G. M. (2011). The generalized inverse Weibull distribution. Statistical Papers 52, 591-619.

25. Haq, M. A., Yousof, H. M. and Hashmi, S. (2017). A New Five-Parameter Fréchet Model for Extreme Values. Pakistan Journal of Statistics and Operation Research, 617-632.

26. Ibrahim, M., Altun, E. and Yousof, H. M. (2020). A new distribution for modeling lifetime data with different methods of estimation and censored regression modeling. Statistics, Optimization \& Information Computing, 8(2), 610-630.

27. Ibrahim. M., Handique, L., Chakraborty, S., Butt, N. S. and M. Yousof, H. (2021). A new three-parameter xgamma Fréchet distribution with different methods of estimation and applications. Pakistan Journal of Statistics and Operation Research, 17(1), 291-308.

28. Ibrahim, M., Yadav, A. S., Yousof, H. M., Goual, H. and Hamedani, G. G. (2019). A new extension of Lindley distribution: modified validation test, characterizations and different methods of estimation. Communications for Statistical Applications and Methods, 26(5), 473-495.

29. Jahanshahi, S.M.A., Yousof, H. M. and Sharma, V.K. (2019). The Burr X Fréchet Model for Extreme Values: Mathematical Properties, Classical Inference and Bayesian Analysis. Pak. J. Stat. Oper. Res., 15(3), 797-818.

30. Korkmaz, M. C., Yousof, H. M. and Ali, M. M. (2017). Some theoretical and computational aspects of the odd Lindley Fréchet distribution. İstatistikçiler Dergisi: İstatistik ve Aktüerya, 10(2), 129-140.

31. Korkmaz, M. C., Yousof, H. M., Hamedani G. G. and Ali, M. M. (2018). The Marshall--Olkin generalized G Poisson family of distributions, Pakistan Journal of Statistics, 34, 251-267.

32. John P. Klein, Melvin L. Moeschberger (1997), Survival Analysis :Techniques for Censored and Truncated Data, Statistics for Biology and Healt.

33. Keller, A. Z. and Kamath, A. R. (1982). Reliability analysis of CNC machine tools. Reliab Eng 3, $449--473$.

34. Kotz, S. and Nadarajah, S. (2000). Extreme value distributions: theory and applications. Imperial College Press, London.

35. Krishna, E., Jose, K. K., Alice, T. and Risti, M. M. (2013). The Marshall-Olkin Fréchet distribution. Communications in Statistics-Theory and Methods, 42, 4091-4107.

36. Mahmoud, M. R. and Mandouh, R. M. (2013). On the transmuted Fréchet distribution. Journal of Applied Sciences Research, 9, 5553-5561.

37. Mansour, M. M., Ibrahim, M., Aidi, K., Shafique Butt, N., Ali, M. M., Yousof, H. M., \& Hamed, M. S. (2020a). A New LogLogistic Lifetime Model with Mathematical Properties, Copula, Modified Goodness-of-Fit Test for Validation and Real Data 
Modeling. Mathematics, 8(9), 1508.

38. Mansour, M. M., Butt, N. S., Ansari, S. I., Yousof, H. M., Ali, M. M., \& Ibrahim, M. (2020b). A new exponentiated Weibull distribution's extension: copula, mathematical properties and applications. Contributions to Mathematics, 1 (2020) 57-66. DOI: $10.47443 / \mathrm{cm} .2020 .0018$

39. Mansour, M., Korkmaz, M. C., Ali, M. M., Yousof, H. M., Ansari, S. I., \& Ibrahim, M. (2020c). A generalization of the exponentiated Weibull model with properties, Copula and application. Eurasian Bulletin of Mathematics, 3(2), 84-102.

40. Mansour, M., Rasekhi, M., Ibrahim, M., Aidi, K., Yousof, H. M., \& Elrazik, E. A. (2020d). A New Parametric Life Distribution with Modified Bagdonavi?ius-Nikulin Goodness-of-Fit Test for Censored Validation, Properties, Applications, and Different Estimation Methods. Entropy, 22(5), 592.

41. Mansour, M., Yousof, H. M., Shehata, W. A., \& Ibrahim, M. (2020e). A new two parameter Burr XII distribution: properties, copula, different estimation methods and modeling acute bone cancer data. Journal of Nonlinear Science and Applications, 13(5), 223-238.

42. Mansour, M. M., Butt, N. S., Yousof, H. M., Ansari, S. I., \& Ibrahim, M. (2020f). A Generalization of Reciprocal Exponential Model: Clayton Copula, Statistical Properties and Modeling Skewed and Symmetric Real Data Sets. Pakistan Journal of Statistics and Operation Research, 16(2), 373-386.

43. Mead, M. E. and Abd-Eltawab A.R. (2014). A note on Kumaraswamy Fréchet distribution. Aust. J. Basic \& Appl. Sci., 8, 294-300.

44. Nadarajah, S. and Kotz, S. (2003). The exponentiated exponential distribution, Satistica, Available online at http://interstat.statjournals.net/YEAR/2003/abstracts/03 12001.php.

45. Nadarajah, S. and Kotz, S. (2008). Sociological models based on Fréchet random variables. Quality and Quantity, $42,89-95$.

46. Nichols, M. D, Padgett, W. J. (2006). A Bootstrap control chart for Weibull percentiles. Quality and Reliability Engineering International, 22, 141-151.

47. Pougaza, D. B. and Djafari, M. A. (2011). Maximum entropies copulas. Proceedings of the 30th international workshop on Bayesian inference and maximum Entropy methods in Science and Engineering, 329-336.

48. Ravi, V. and Gilbert, P. D. (2009), BB: An R package for solving a large system of nonlinear equations and for optimizing a high-dimensional nonlinear objective function, J. Statist. Software, 32(4).

49. Rodriguez-Lallena, J. A. and Ubeda-Flores, M. (2004). A new class of bivariate copulas. Statistics and Probability Letters, $66,315--25$.

50. Salah, M. M., El-Morshedy, M., Eliwa, M. S. and Yousof, H. M. (2020). Expanded Fréchet Model: Mathematical Properties, Copula, Different Estimation Methods, Applications and Validation Testing. Mathematics, 8(11), 1949.

51. Shehata, W. A. M. and Yousof, H. M. (2021a). A novel two-parameter Nadarajah-Haghighi extension: properties, copulas, modeling real data and different estimation methods. Statistics, Optimization \& Information Computing, forthcoming.

52. Shehata, W. A. M. and Yousof, H. M. (2021b). The four-parameter exponentiated Weibull model with Copula, properties and real data modeling. Pakistan Journal of Statistics and Operation Research, 17(3), 649-667.

53. Shahbaz, S. H., Ismail, M. and Butt, N. S. (2016). The McDonald's inverse Weibull distribution. Pak. J. Stat. Oper. Res. Vol. XII (4), 775-785

54. Smith, R. L. and Naylor, J. C. (1987). A comparison of maximum likelihood and bayesian estimators for the three-parameter Weibull distribution. Applied Statistics, 36, 358-369.

55. Wright, E. M. (1935). The asymptotic expansion of the generalized hypergeometric function. Journal of the London Mathematical Society, 10, 286-293.

56. Yadav, A. S., Goual, H., Alotaibi, R. M., Ali, M. M. and Yousof, H. M. (2020). Validation of the Topp-Leone-Lomax model via a modified Nikulin-Rao-Robson goodness-of-fit test with different methods of estimation. Symmetry, 12(1), 57.

57. Yousof, H. M., Altun, E. and Hamedani, G. G. (2018a). A new extension of Frechet distribution with regression models, residual analysis and characterizations. Journal of Data Science, 16(4), 743-770.

58. Yousof, H. M., Altun, E., Rasekhi, M., Alizadeh, M., Hamedani, G. G. and Ali, M. M. (2019a). A new lifetime model with regression models, characterizations and applications. Communications in Statistics-Simulation and Computation, 48(1), 264286.

59. Yousof, H. M., Alizadeh, M., Jahanshahiand, S. M. A., Ramires, T. G., Ghosh, I. and Hamedani G. G. (2017). The transmuted Topp-Leone $\mathrm{G}$ family of distributions: theory, characterizations and applications, Journal of Data Science. 15, 723-740.

60. Yousof, H. M., Butt, N. S., Alotaibi, R. M., Rezk, H., Alomani, G. A., \& Ibrahim, M. (2019b). A new compound Fréchet distribution for modeling breaking stress and strengths data. Pakistan Journal of Statistics and Operation Research, 15(4), 1017-1035.

61. Yousof, H. M., Hamedani, G. G., \& Ibrahim, M. (2020). The Two-parameter Xgamma Fréchet Distribution: Characterizations, Copulas, Mathematical Properties and Different Classical Estimation Methods. Contributions to Mathematics, 2 (2020), 3241.

62. Yousof, H. M., Jahanshahi, S. M. A., Ramires, T. G., Aryal, G. R., \& Hamedani, G. G. (2018b). A new distribution for extreme values: regression model, characterizations and applications. Journal of Data Science, 16(4), 677 -706.

63. Yousof, H. M., Rasekhi, M., Altun, E., \& Alizadeh, M. (2018). The extended odd Fréchet family of distributions: properties, applications and regression modeling. International Journal of Applied Mathematics and Statistics, 30(1), 1-30.

64. Zaharim, A., Najid, S.K., Razali, A.M. and Sopian, K. (2009). Analysing Malaysian wind speed data using statistical distribution. In Proceedings of the 4th IASME/WSEAS International conference on energy and environment, Cambridge, UK. 